\title{
Research Article \\ Zeros of the Exceptional Laguerre and Jacobi Polynomials
}

\author{
Choon-Lin Ho ${ }^{1}$ and Ryu Sasaki ${ }^{2}$ \\ ${ }^{1}$ Department of Physics, Tamkang University, Tamsui 251, Taiwan \\ ${ }^{2}$ Yukawa Institute for Theoretical Physics, Kyoto University, Kyoto 606-8502, Japan \\ Correspondence should be addressed to Choon-Lin Ho, hcl@mail.tku.edu.tw
}

Received 12 April 2012; Accepted 4 July 2012

Academic Editors: G. Goldin and R. Schiappa

Copyright (C) 2012 C.-L. Ho and R. Sasaki. This is an open access article distributed under the Creative Commons Attribution License, which permits unrestricted use, distribution, and reproduction in any medium, provided the original work is properly cited.

An interesting discovery in the last two years in the field of mathematical physics has been the exceptional $X_{\ell}$ Laguerre and Jacobi polynomials. Unlike the well-known classical orthogonal polynomials which start with constant terms, these new polynomials have the lowest degree $\ell=1,2, \ldots$, and yet they form complete sets with respect to some positive-definite measure. In this paper, we study one important aspect of these new polynomials, namely, the behaviors of their zeros as some parameters of the Hamiltonians change. Most results are of heuristic character derived by numerical analysis.

\section{Introduction}

The discovery of new types of orthogonal polynomials, called the exceptional $X_{\ell}$ polynomials, has been the most interesting development in the area of exactly solvable models in quantum mechanics in the last two years [1-10]. Unlike the classical orthogonal polynomials, these new polynomials have the remarkable properties that they still form complete sets with respect to some positive-definite measure, although they start with degree $\ell$ polynomials instead of a constant. Four sets of infinite families of such polynomials, namely, the Laguerre type L1, L2, and the Jacobi type J1, J2 $X_{\ell}$ polynomials, with $\ell=1,2, \ldots$, were constructed in [3-5]. These systems were derived by deforming the radial oscillator potential and the Darboux-Pöschl-Teller (DPT) potential in terms of an eigen polynomial of degree $\ell(\ell=1,2, \ldots)$. The lowest $(\ell=1)$ examples, the $X_{1}$-Laguerre and $X_{1}$-Jacobi polynomials, are equivalent to those introduced in the pioneering work of Gomez-Ullate et al. $[1,2]$ within the Sturm-Liouville theory. The results in $[1,2]$ were reformulated in the framework of quantum mechanics and shape-invariant potentials by Quesne et al. [6-8]. 
By construction, these new orthogonal polynomials satisfy a second order differential equation (the Schrödinger equation) without contradicting Bochner's theorem [11], since they start at degree $\ell>0$ instead of the degree zero constant term. Generalization of exceptional orthogonal polynomials to discrete quantum mechanical systems was done in $[12,13]$.

Later, equivalent but much simpler looking forms of the Laguerre- and Jacobi-type $X_{\ell}$ polynomials than those originally presented in [3-5] were given in [9]. These nice forms were derived based on an analysis of the second order differential equations for the $X_{\ell}$ polynomials within the framework of the Fuchsian differential equations in the entire complex $x$-plane. They allow us to study in depth some important properties of the $X_{\ell}$ polynomials, such as the actions of the forward and backward shift operators on the $X_{\ell}$ polynomials, Gram-Schmidt orthonormalization for the algebraic construction of the $X_{\ell}$ polynomials, Rodrigues formulas, and the generating functions of these new polynomials.

Recently, these exceptional orthogonal polynomials were generated by means of the Darboux-Crum transformation [14, 15]. Physical models which may involve these new polynomials were considered in [16].

One important aspect related to these new polynomials, which was only briefly mentioned in [9] but has not been investigated in depth so far, is the structure of their zeros. It is the purpose of this paper to look into this. Particularly, we investigate the behaviors of the zeros as the parameters of the polynomials change through numerical analysis.

The plan of this paper is as follows. In Section 2, we briefly review the forms of the exceptional polynomials. Sections 3 and 4 study the behaviors of the extra and the ordinary zeros, respectively, of the exceptional polynomials as one of $\ell$ and $n$ increases while the other parameters being kept fixed. Section 5 presents analytical proofs that explain the movements of the extra zeros of the exceptional polynomials as $n$ changes at fixed $\ell$. In Section 6, we consider behaviors of the zeros at large $g$ and/or $h$. Section 7 summarizes the paper. In the Appendix, we list a few lower degree exceptional orthogonal polynomials for reference. We do believe these heuristic results exemplify the essential features of the zeros of the exceptional orthogonal polynomials and that they would instigate more rigorous research of this novel and interesting subject.

\section{Exceptional Orthogonal Polynomials}

Four sets of infinitely many exceptional orthogonal polynomials were derived in [3$5]$, among them two are deformations of the Laguerre polynomials, and the others are deformations of the Jacobi polynomials. A unified nice form of these polynomials was given in [9], in which these polynomials are expressed as a bilinear form of the original polynomials, the Laguerre or Jacobi polynomials and the deforming polynomials, depending on the set of parameters $\mathcal{\lambda}$ and their shifts $\delta$ and a nonnegative integer $\ell$, which is the degree of the deforming polynomials. The two sets of exceptional Laguerre polynomials $(\ell=1,2, \ldots$, $n=0,1,2, \ldots)$ are [9]

$$
P_{\ell, n}(\eta ; \boldsymbol{l}) \stackrel{\text { def }}{=}\left\{\begin{array}{cc}
\xi_{\ell}(\eta ; \boldsymbol{\lambda}+\boldsymbol{\delta}) P_{n}(\eta ; g+\ell-1)-\xi_{\ell}(\eta ; \boldsymbol{l}) \partial_{\eta} P_{n}(\eta ; g+\ell-1) & : \mathrm{L} 1 \\
\left(n+g+\frac{1}{2}\right)^{-1}\left\{\begin{array}{c}
\left(g+\frac{1}{2}\right) \xi_{\ell}(\eta ; \boldsymbol{\lambda}+\boldsymbol{\delta}) P_{n}(\eta ; g+\ell+1) \\
\left.+\eta \xi_{\ell}(\eta ; \boldsymbol{l}) \partial_{\eta} P_{n}(\eta ; g+\ell+1)\right\}
\end{array}\right. & : \text { L2 }
\end{array}\right.
$$


in which $\mathcal{l} \stackrel{\text { def }}{=} g>0, \delta \stackrel{\text { def }}{=} 1$ and

$$
P_{n}(\eta ; g) \stackrel{\text { def }}{=} L_{n}^{(g-(1 / 2))}(\eta), \quad \xi_{\ell}(\eta ; g) \stackrel{\text { def }}{=} \begin{cases}L_{\ell}^{(g+\ell-(3 / 2))}(-\eta) & : \mathrm{L} 1 \\ L_{\ell}^{(-g-\ell-(1 / 2))}(\eta) & : \text { L2 } .\end{cases}
$$

Here $L_{n}^{(\alpha)}(\eta)$ are the classical Laguerre polynomials (A.1). It is interesting to note the following alternative expressions for the L1 and L2 exceptional polynomials:

$$
P_{\ell, n}(\eta ; \lambda) \stackrel{\text { def }}{=}\left\{\begin{array}{cc}
L_{\ell}^{(g+\ell-(1 / 2))}(-\eta) L_{n}^{(g+\ell-(1 / 2))}(\eta)-L_{\ell-1}^{(g+\ell-(1 / 2))}(-\eta) L_{n-1}^{(g+\ell-(1 / 2))}(\eta) & : \mathrm{L} 1 \\
(n+g+(1 / 2))^{-1}\left\{\left(g+\left(\frac{1}{2}\right)\right) L_{\ell}^{(-g-\ell-(3 / 2))}(\eta) L_{n}^{(g+\ell+(3 / 2))}(\eta)\right. & \\
\left.+(\ell+1) L_{\ell+1}^{(-g-\ell-(3 / 2))}(\eta) L_{n-1}^{(g+\ell+(3 / 2))}(\eta)\right\} & : \mathrm{L} 2 .
\end{array}\right.
$$

The two sets of exceptional Jacobi polynomials $(\ell=1,2, \ldots, n=0,1,2, \ldots)$ are [9]

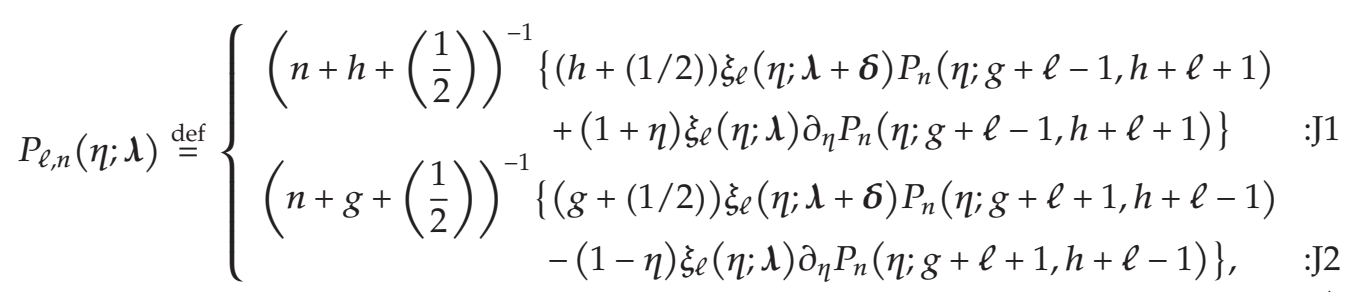

in which $\mathcal{\lambda} \stackrel{\text { def }}{=}(g, h), g>0, h>0, \delta \stackrel{\text { def }}{=}(1,1)$ and

$$
P_{n}(\eta ; g, h) \stackrel{\text { def }}{=} P_{n}^{(g-(1 / 2), h-(1 / 2))}(\eta), \xi_{\ell}(\eta ; g, h) \stackrel{\text { def }}{=} \begin{cases}P_{\ell}^{(g+\ell-(3 / 2),-h-\ell-(1 / 2))}(\eta), & g>h>0: \mathrm{J} 1 \\ P_{\ell}^{(-g-\ell-(1 / 2), h+\ell-(3 / 2))}(\eta), & h>g>0,: \mathrm{J} 2,\end{cases}
$$

where $P_{n}^{(\alpha, \beta)}(\eta)$ are the classical Jacobi polynomials (A.2). The new exceptional orthogonal polynomials can be viewed as deformations of the classical orthogonal polynomials by the parameter $\ell$, and the two polynomials $\xi_{\ell}(\eta ; \lambda)$ and $\xi_{\ell}(\eta ; \lambda+\delta)$ played the role of the deforming polynomials. 
The differential equations satisfied by these new polynomials are as follows:

$$
\begin{aligned}
& \mathrm{L} 1: \eta \partial_{\eta}^{2} P_{\ell, n}(\eta ; \lambda)+\left(g+\ell+\frac{1}{2}-\eta-2 \frac{\eta \partial_{\eta} \xi_{\ell}(\eta ; \lambda)}{\xi_{\ell}(\eta ; \lambda)}\right) \partial_{\eta} P_{\ell, n}(\eta ; \lambda) \\
& +\left(2 \frac{\eta \partial_{\eta} \xi_{\ell}(\eta ; \boldsymbol{\lambda}+\boldsymbol{\delta})}{\xi_{\ell}(\eta ; \boldsymbol{\lambda})}+n-\ell\right) P_{\ell, n}(\eta ; \boldsymbol{\lambda})=0, \\
& \mathrm{~L} 2: \eta \partial_{\eta}^{2} P_{\ell, n}(\eta ; \mathcal{\lambda})+\left(g+\ell+\frac{1}{2}-\eta-2 \frac{\eta \partial_{\eta} \xi_{\ell}(\eta ; \lambda)}{\xi_{\ell}(\eta ; \lambda)}\right) \partial_{\eta} P_{\ell, n}(\eta ; \lambda) \\
& +\left(-2 \frac{(g+(1 / 2)) \partial_{\eta} \xi_{\ell}(\eta ; \boldsymbol{\lambda}+\boldsymbol{\delta})}{\xi_{\ell}(\eta ; \boldsymbol{\lambda})}+n+\ell\right) P_{\ell, n}(\eta ; \boldsymbol{\lambda})=0, \\
& \mathrm{~J} 1:\left(1-\eta^{2}\right) \partial_{\eta}^{2} P_{\ell, n}(\eta ; \lambda)+\left(h-g-(g+h+2 \ell+1) \eta-2 \frac{\left(1-\eta^{2}\right) \partial_{\eta} \xi_{\ell}(\eta ; \lambda)}{\xi_{\ell}(\eta ; \lambda)}\right) \partial_{\eta} P_{\ell, n}(\eta ; \boldsymbol{\lambda}) \\
& +\left(-\frac{2(h+(1 / 2))(1-\eta) \partial_{\eta} \xi_{\ell}(\eta ; \boldsymbol{\lambda}+\boldsymbol{\delta})}{\xi_{\ell}(\eta ; \boldsymbol{\lambda})}+\ell(\ell+g-h-1)\right. \\
& +n(n+g+h+2 \ell)) P_{\ell, n}(\eta ; \lambda)=0, \\
& \mathrm{~J} 2:\left(1-\eta^{2}\right) \partial_{\eta}^{2} P_{\ell, n}(\eta ; \lambda)+\left(h-g-(g+h+2 \ell+1) \eta-2 \frac{\left(1-\eta^{2}\right) \partial_{\eta} \xi_{\ell}(\eta ; \lambda)}{\xi_{\ell}(\eta ; \lambda)}\right) \partial_{\eta} P_{\ell, n}(\eta ; \lambda) \\
& +\left(\frac{2(g+(1 / 2))(1+\eta) \partial_{\eta} \xi_{\ell}(\eta ; \boldsymbol{\lambda}+\delta)}{\xi_{\ell}(\eta ; \mathcal{l})}+\ell(\ell+h-g-1)\right. \\
& +n(n+g+h+2 \ell)) P_{\ell, n}(\eta ; \lambda)=0 .
\end{aligned}
$$

The zeros of orthogonal polynomials have always attracted the interest of researchers. In this paper, we will study the properties of the zeros of these new exceptional polynomials as some of their basic parameters change, mainly by numerical analysis.

In the case of $X_{\ell}$ polynomial $P_{\ell, n}(\eta ; \mathcal{l})$, it has $n$ zeros in the (ordinary) domain, where the weight function is defined, that is, $(0, \infty)$ for the L1 and L2 polynomials and $(-1,1)$ for the $\mathrm{J} 1$ and J2 polynomials. The behavior of these zeros, which we will call the ordinary zeros, is the same as those of other ordinary orthogonal polynomials [17-20]. We will say more about these zeros in Section 4 . Besides these $n$ zeros, there are extra $\ell$ zeros outside the ordinary 
domain. For convenience, we will adopt the following notation for the zeros of the various polynomials involved:

$$
\begin{gathered}
\bar{\xi}_{k}^{(\ell)}: \text { zeros of } \xi_{\ell}(\eta ; \boldsymbol{l}+\boldsymbol{\delta}), \quad k=1,2, \ldots, \ell ; \\
\xi_{k}^{(\ell)}: \text { zeros of } \xi_{\ell}(\eta ; \mathcal{l}), \quad k=1,2, \ldots, \ell ; \\
\bar{\eta}_{k}^{(\ell, n)}: \text { extra zeros of } P_{\ell, n}, \quad k=1,2, \ldots, \ell ; \\
\eta_{j}^{(\ell, n)}: \text { ordinary zeros of } P_{\ell, n,} \quad j=1,2, \ldots, n .
\end{gathered}
$$

We emphasize that $\eta_{j}^{(\ell, n)} \in(0, \infty)$ for the L1 and L2 Laguerre polynomials, and $\eta_{j}^{(\ell, n)} \in(-1,1)$ for the J1 and J2 Jacobi polynomials.

Figures 1-10 depict the distribution of the zeros for some representative parameters of the systems, namely, $n, l, g$, and $h$. From these figures, one can deduce certain patterns of the distribution of the zeros as those parameters vary. We will discuss these behaviors below.

\section{Extra $\ell$ Zeros of $X_{\ell}$ Polynomials}

Here we discuss the locations of the extra $\ell$ zeros of the exceptional orthogonal polynomials, which lie in different positions for the different types of polynomials. From our numerical analysis, we can summarize the trend as follows.

The $\ell$ extra zeros of $\mathrm{L} 1$ polynomials are on the negative real line $(-\infty, 0)$. The $\mathrm{L} 2 \mathrm{X}_{\ell \text { :odd }}$ polynomials have one real negative zero which lies to the left of the remaining $(1 / 2)(\ell-1)$ pairs of complex conjugate roots. The $\mathrm{L} 2 X_{\ell: \text { even }}$ polynomials have $(1 / 2) \ell$ pairs of complex conjugate roots.

The situations for the $X_{\ell}$ Jacobi polynomials are a bit more complicated. The J1 $X_{\ell \text { :odd }}$ polynomials have one real negative root which lies to the left of the remaining $(1 / 2)(\ell-1)$ pairs of complex conjugate roots with negative real parts. The J1 $X_{\ell: e v e n}$ polynomials have $(1 / 2) \ell$ pairs of complex conjugate roots with negative real parts. The J2 $X_{\ell \text { :odd }}$ polynomials have one real positive root which lies to the right of the remaining $(1 / 2)(\ell-1)$ pairs of complex conjugate roots with positive real parts. The $\mathrm{J} 2 \mathrm{X}_{\ell \text { :even }}$ polynomials have $(1 / 2) \ell$ pairs of complex conjugate roots with positive real parts.

One notes that the J1 and J2 polynomials are the mirror images of each other, in the sense $\eta \leftrightarrow-\eta$ and $g \leftrightarrow h$, as exemplified by the relation $\xi_{\ell}^{]^{2}}(\eta ; g, h)=(-1)^{\ell} \xi_{\ell}{ }^{1}(-\eta ; h, g)[3-$ $5,9]$. So the behaviors of the zeros of J1 Jacobi polynomials can be obtained from those of the J2 type accordingly. As such, for clarity of presentation, we will only discuss the behaviors of the zeros of the J2 Jacobi polynomials in this paper. 

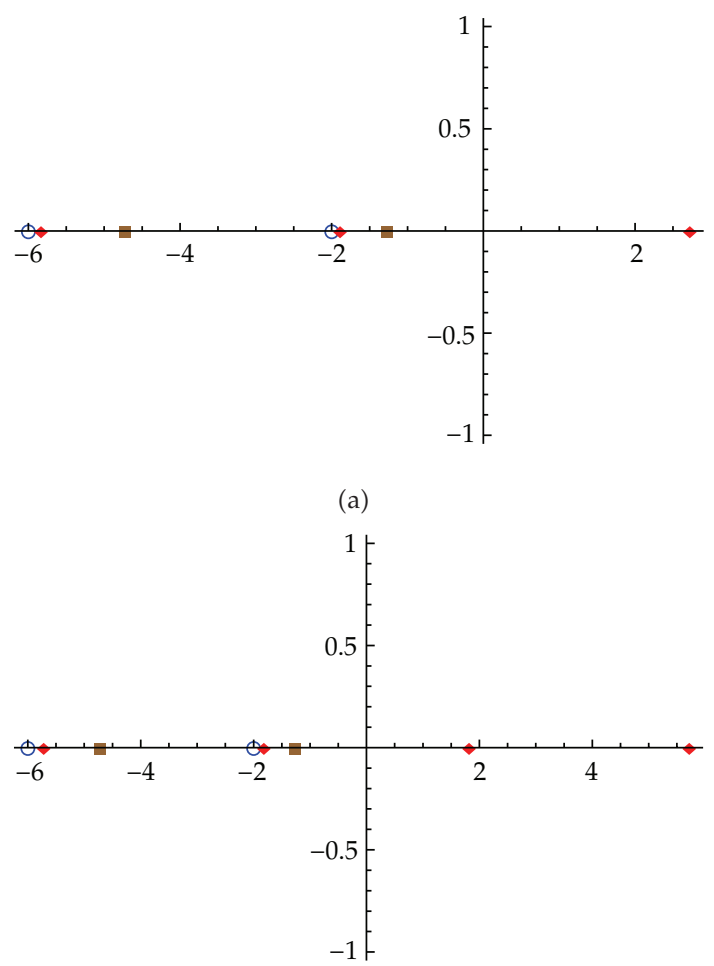

(b)

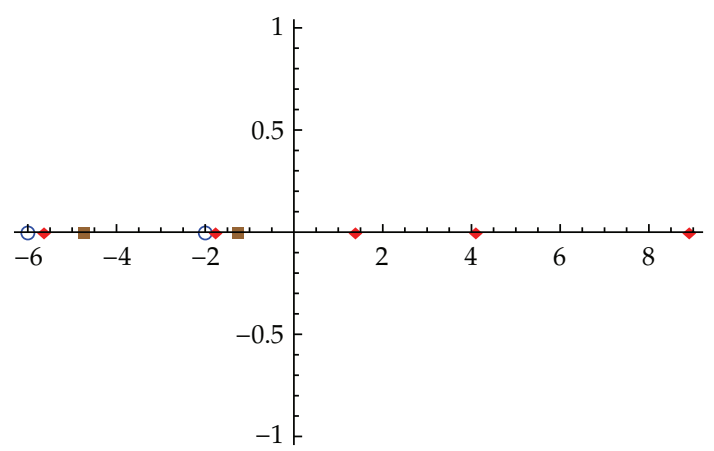

(c)

Figure 1: L1: distributions of the zeros $\bar{\eta}_{k}^{(\ell, n)}, \eta_{j}^{(\ell, n)}(\bullet), \bar{\xi}_{k}^{(\ell)}(\bigcirc)$, and $\xi_{k}^{(\ell)}(\boldsymbol{\square})$ for the L1 Laguerre polynomials, with $g=0.5$ and $\ell=2$. The three diagrams correspond to $n=1(\mathrm{a}), 2(\mathrm{~b})$, and $3(\mathrm{c})$, respectively. The ordinary zeros $\eta_{j}^{(\ell, n)}$ lie in $(0, \infty)$.

\subsection{Behaviors as $n$ Increases at Fixed $\ell$}

In all cases, we have

$$
P_{\ell, 0}(\eta ; \lambda) \propto \xi_{\ell}(\eta ; \boldsymbol{\lambda}+\delta)
$$

This implies that the zeros of $P_{\ell, 0}$ coincide with those of $\xi_{\ell}(\eta ; \boldsymbol{\lambda}+\boldsymbol{\delta})$, namely, $\bar{\xi}_{k}^{(\ell)}, k=1,2, \ldots, \ell$. 


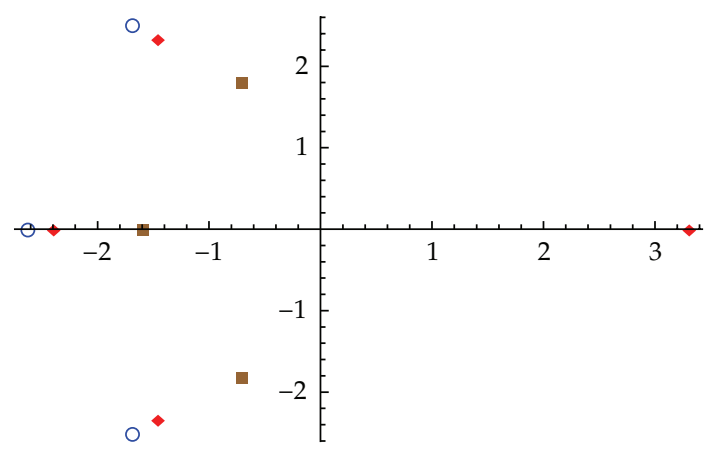

(a)

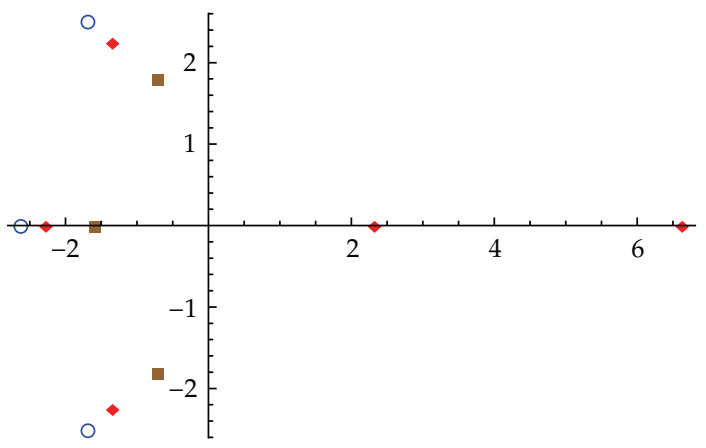

(b)

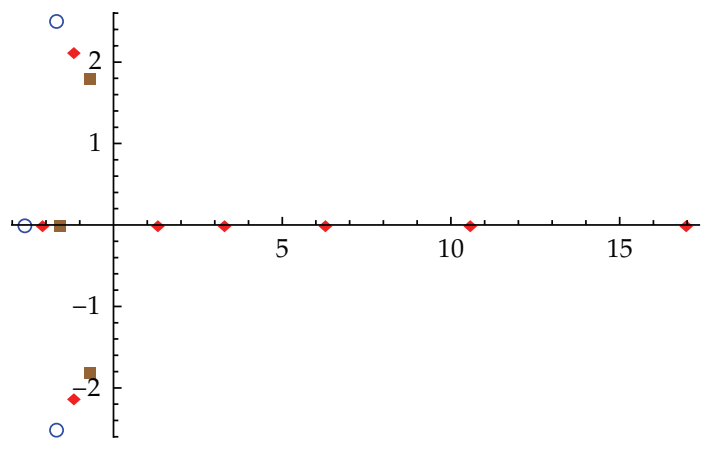

(c)

Figure 2: L2: distributions of the zeros $\bar{\eta}_{k}^{(\ell, n)}, \eta_{j}^{(\ell, n)}(\diamond), \bar{\xi}_{k}^{(\ell)}(\bigcirc)$, and $\xi_{k}^{(\ell)}(\boldsymbol{\square})$ for the L2 Laguerre polynomials, with $g=0.5$ and $\ell=3$. The three diagrams correspond to $n=1(\mathrm{a}), 2(\mathrm{~b})$, and $5(\mathrm{c})$, respectively. The ordinary zeros $\eta_{j}^{(\ell, n)}$ lie in $(0, \infty)$.

At fixed $\ell$, all the $\bar{\eta}_{k}^{(\ell, n)}$ move from $\bar{\xi}_{k}^{(\ell)}$ at $n=0$ to $\xi_{k}^{(\ell)}$ as $n \rightarrow \infty$. This can be seen from Figures 1-3 and in Tables 1-7. We will provide heuristic arguments for this result in Section 5. 


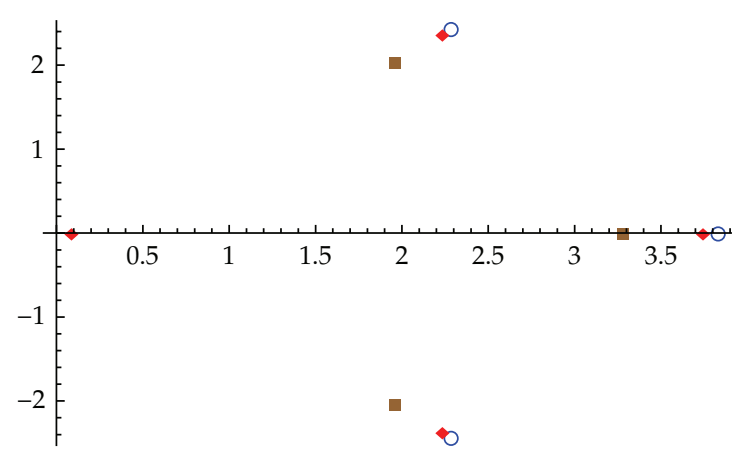

(a)

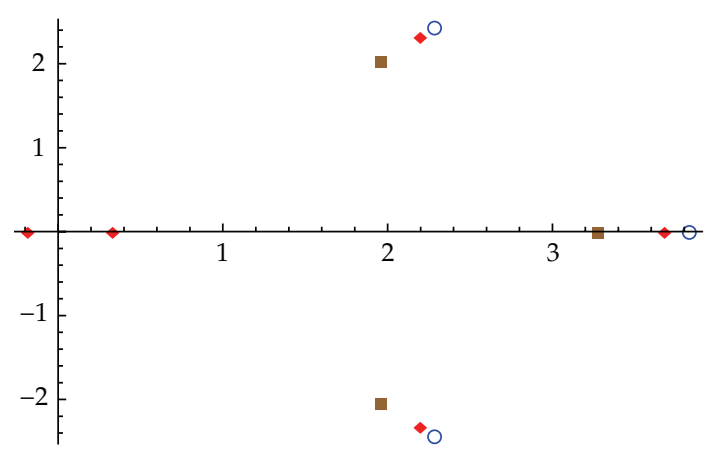

(b)

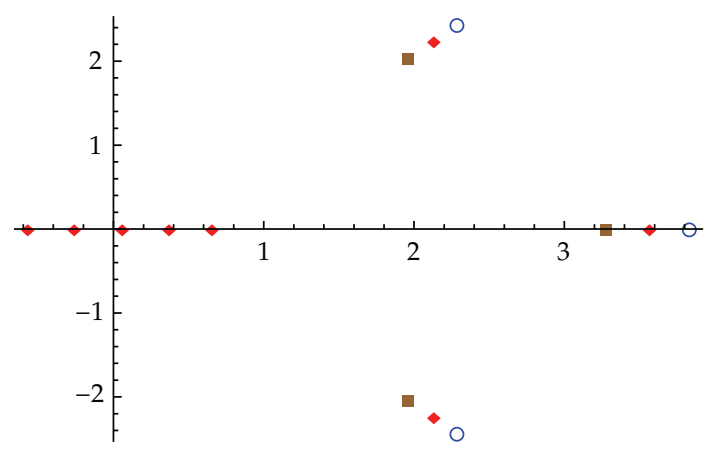

(c)

Figure 3: J2: distributions of the zeros $\bar{\eta}_{k}^{(\ell, n)}, \eta_{j}^{(\ell, n)}(\bullet), \bar{\xi}_{k}^{(\ell)}(\bigcirc)$, and $\xi_{k}^{(\ell)}(\boldsymbol{\square})$ for the J2 Jacobi polynomials, with $g=3, h=4$, and $\ell=3$. The three diagrams correspond to $n=1(\mathrm{a}), 2(\mathrm{~b})$, and $5(\mathrm{c})$, respectively. The ordinary zeros $\eta_{j}^{(\ell, n)}$ lie in $(-1,1)$.

\subsection{Behaviors as $\ell$ Increases at Fixed $n$}

The discussions in the last subsection show that $\bar{\eta}_{k}^{(\ell, n)}$ by proper numbering are sandwiched between $\bar{\xi}_{k}^{(\ell)}$ and $\xi_{k}^{(\ell)}$. Thus to know how $\bar{\eta}_{k}^{(\ell, n)}$ behave as $\ell$ increases at fixed $n$, we only need to study how the zeros $\bar{\xi}_{k}^{(\ell)}$ and $\xi_{k}^{(\ell)}$ flow as $\ell$ increases. 


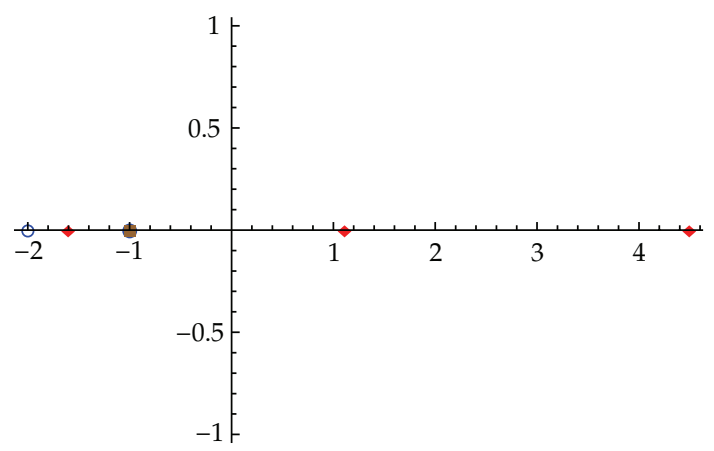

(a)

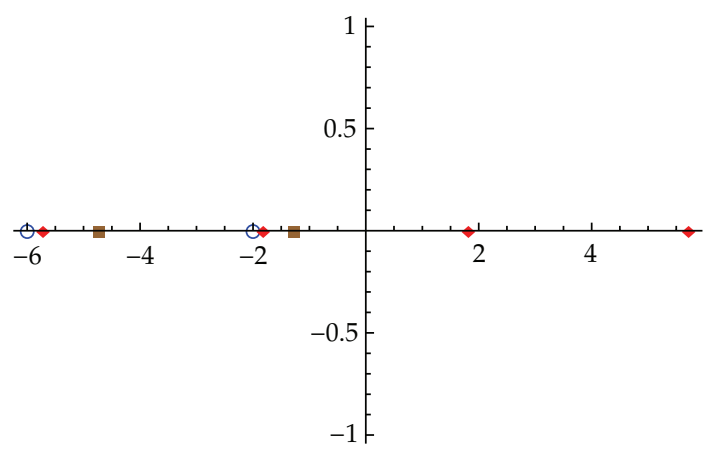

(b)

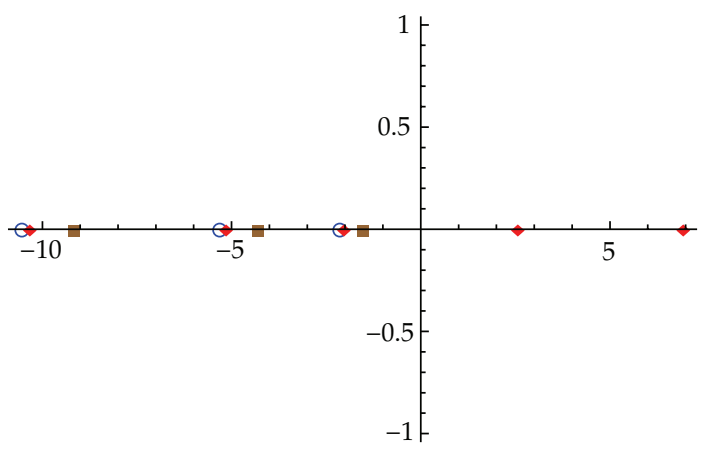

(c)

Figure 4: L1: distributions of the zeros $\bar{\eta}_{k}^{(\ell, n)}, \eta_{j}^{(\ell, n)}(\downarrow), \bar{\xi}_{k}^{(\ell)}(\bigcirc)$, and $\xi_{k}^{(\ell)}(\mathbb{\square})$ for the L1 Laguerre polynomials, with $g=0.5$ and $n=2$. The three diagrams correspond to $\ell=1(\mathrm{a}), 2(\mathrm{~b})$, and 3(c), respectively. 


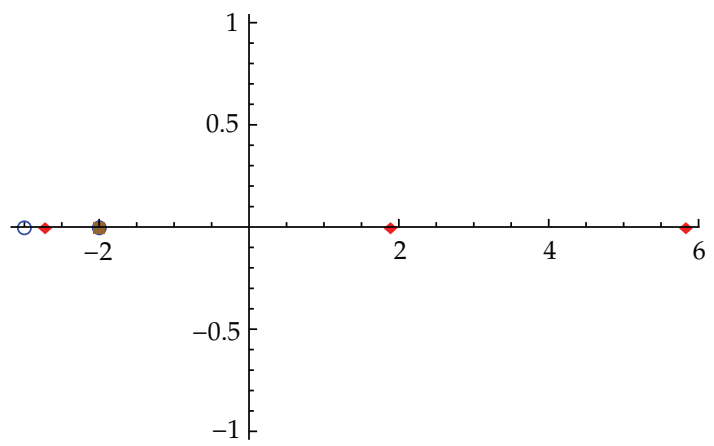

(a)

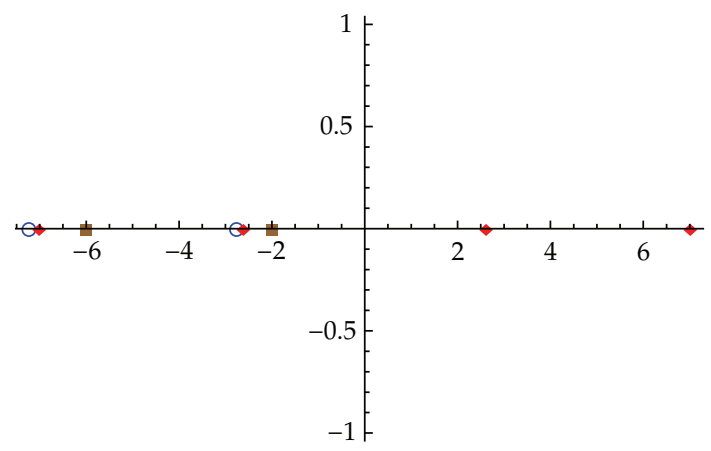

(b)

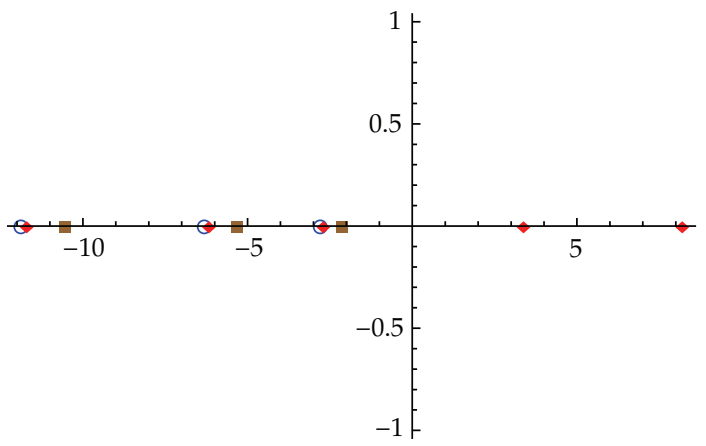

(c)

Figure 5: L1: distributions of the zeros $\bar{\eta}_{k}^{(\ell, n)}, \eta_{j}^{(\ell, n)}(\bullet), \bar{\xi}_{k}^{(\ell)}(\bigcirc)$, and $\xi_{k}^{(\ell)}(\boldsymbol{\square})$ for the L1 Laguerre polynomials, with $g=1.5$ and $n=2$. The three diagrams correspond to $\ell=1(\mathrm{a}), 2(\mathrm{~b})$, and 3(c), respectively. 


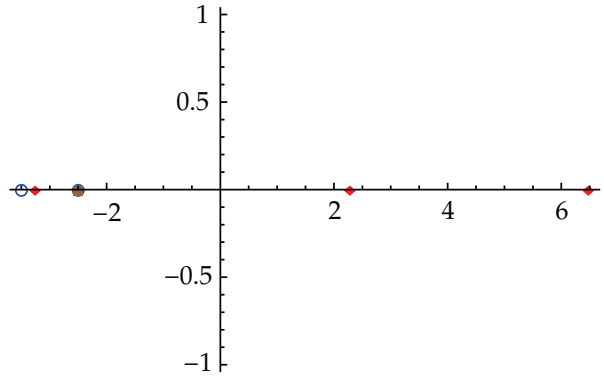

(a)

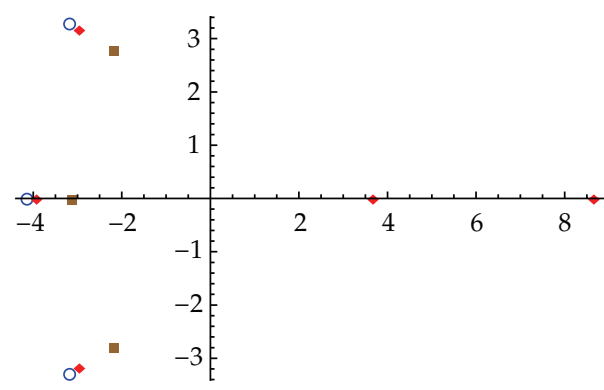

(c)

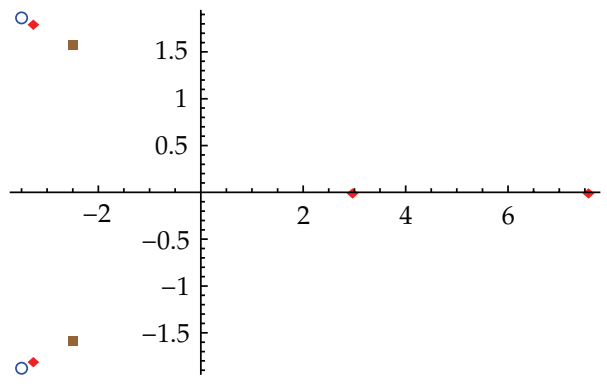

(b)

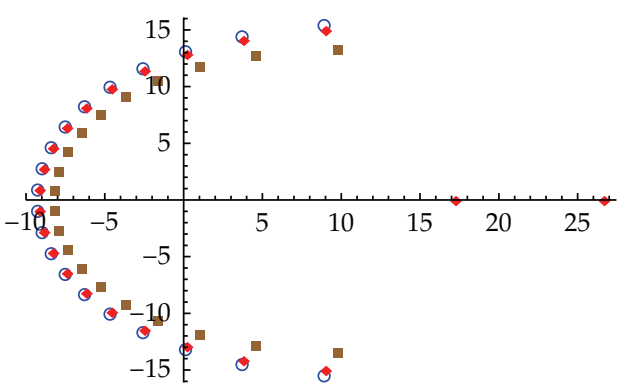

(d)

Figure 6: L2: distributions of the zeros $\bar{\eta}_{k}^{(\ell, n)}, \eta_{j}^{(\ell, n)}(\diamond), \bar{\xi}_{k}^{(\ell)}(\bigcirc)$, and $\xi_{k}^{(\ell)}(\mathbf{\square})$ for the L2 Laguerre polynomials, with $g=2$ and $n=2$. The four diagrams correspond to $\ell=1(\mathrm{a}), 2(\mathrm{~b}), 3(\mathrm{c})$, and 20(d), respectively.

\subsubsection{L1 Laguerre}

As $\ell$ changes to $\ell+1$, the zeros of $\xi_{\ell}(\eta ; g+1)$ and $\xi_{\ell}(\eta ; g)$ decrease (move to the left), and a new set of zeros appear from the right.

$$
\begin{gathered}
\bar{\xi}_{k}^{(\ell+1)}<\bar{\xi}_{k}^{(\ell)}, \\
\xi_{k}^{(\ell+1)}<\xi_{k}^{(\ell)}, \\
\bar{\xi}_{k}^{(\ell)}<\xi_{k}^{(\ell)}<\bar{\xi}_{k+1}^{(\ell)}<\xi_{k+1}^{(\ell)},
\end{gathered}
$$

for $k=1,2, \ldots, \ell-1, \ell$.

We show these patterns for some representative parameters in Figures 4 and 5.

\subsubsection{L2 Laguerre}

For $\ell=1$, there is one real root each for $\xi_{\ell}(\eta ; g+1)$ and $\xi_{\ell}(\eta ; g)$, with $\bar{\xi}_{1}^{(\ell)}<\xi_{1}^{(\ell)}<0$.

For $\ell=2$, the above two roots bifurcate into two complex roots, with $\mathfrak{R} \bar{\xi}^{(\ell)}<\mathfrak{R} \xi^{(\ell)}$, $\left|\Im \xi^{(\ell)}\right|>\left|\Im \xi^{(\ell)}\right|$. 


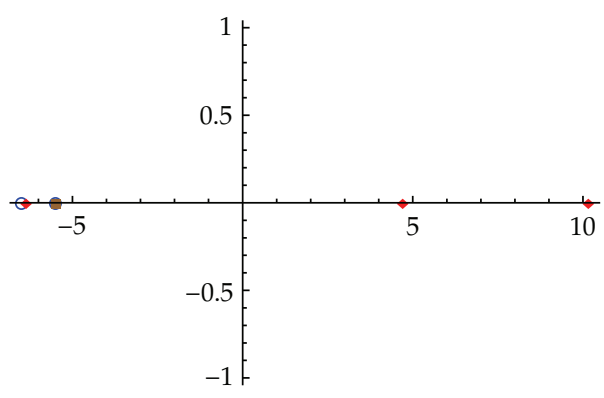

(a)

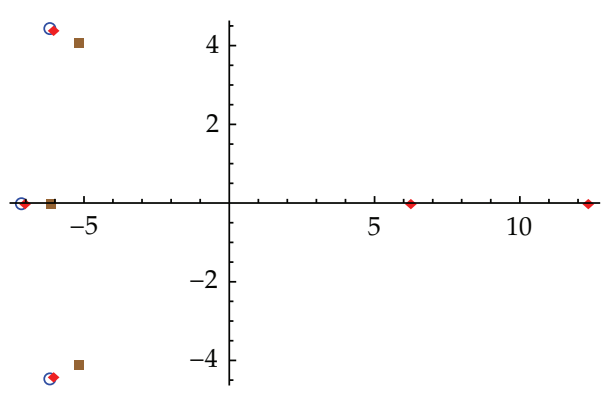

(c)

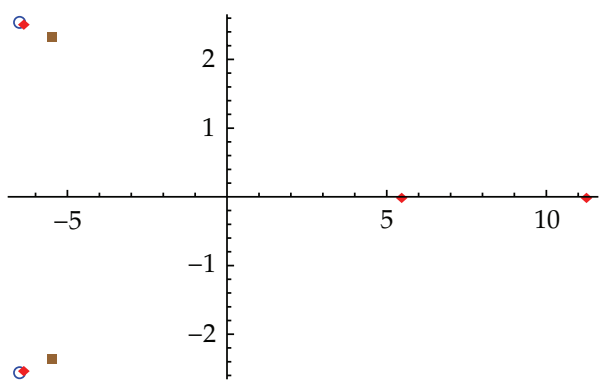

(b)

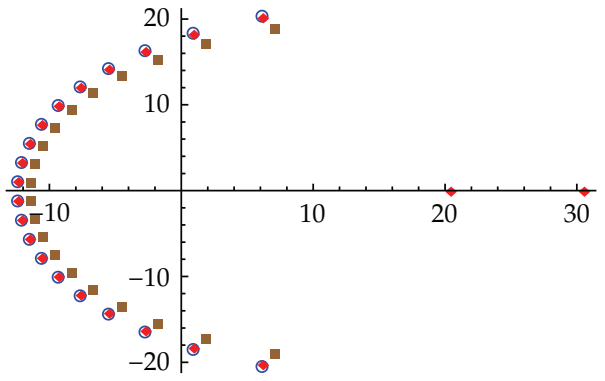

(d)

Figure 7: L2: distributions of the zeros $\bar{\eta}_{k}^{(\ell, n)}, \eta_{j}^{(\ell, n)}(\diamond), \bar{\xi}_{k}^{(\ell)}(\bigcirc)$, and $\xi_{k}^{(\ell)}(\boldsymbol{\square})$ for the L2 Laguerre polynomials, with $g=5$ and $n=2$. The four diagrams correspond to $\ell=1(\mathrm{a}), 2(\mathrm{~b}), 3(\mathrm{c})$, and 20(d), respectively.

Generally, for even $\ell$, there are $\ell$ complex zeros with

$$
\mathfrak{R} \bar{\xi}_{k}^{(\ell)}<\mathfrak{R} \xi_{k}^{(\ell)},\left|\Im \bar{\xi}_{k}^{(\ell)}\right|>\left|\Im \xi_{k}^{(\ell)}\right|, \quad k=1,2, \ldots, \frac{\ell}{2}
$$

All $\bar{\eta}_{k}^{(\ell, n)}$ are sandwiched between $\bar{\xi}_{k}^{(\ell)}$ and $\xi_{k}^{(\ell)}$. As an even $\ell$ changes to $\ell+1$ which is odd, all zeros move to the right with the real and the absolute value of the imaginary parts increased, and a new real zero appears to the left of all the complex zeros on the negative real axis. As $\ell$ increases further, the complex zeros move as described before, and the zero on the negative real axis bifurcates into two complex zeros, giving an even number of complex zeros. These patterns continue as $\ell$ increases.

Figures 6 and 7 show these behaviors for some selected parameters. For large $\ell$, these zeros distribute in a horse-shoe pattern.

\subsubsection{J2 Jacobi}

For $\ell=1$, there is one real root each for $\xi_{\ell}(\eta ; g+1)$ and $\xi_{\ell}(\eta ; g)$, with $\bar{\xi}_{1}^{(\ell)}>\xi_{1}^{(\ell)}>1$.

For $\ell=2$, the above two roots bifurcate into two complex roots, with $\mathfrak{R}^{-(\ell)}>\Re \xi^{(\ell)}$, $\left|\Im \xi^{(\ell)}\right|>\left|\Im \xi^{(\ell)}\right|$. 


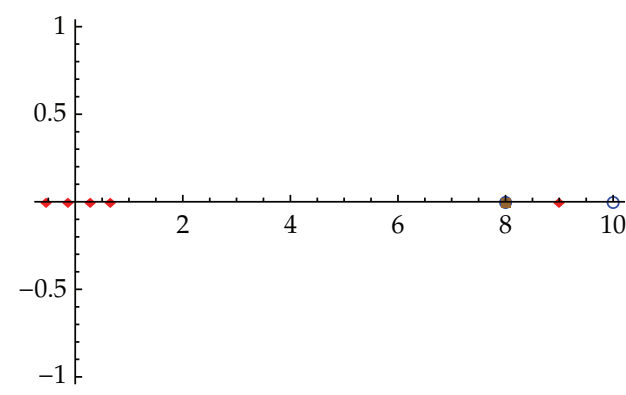

(a)

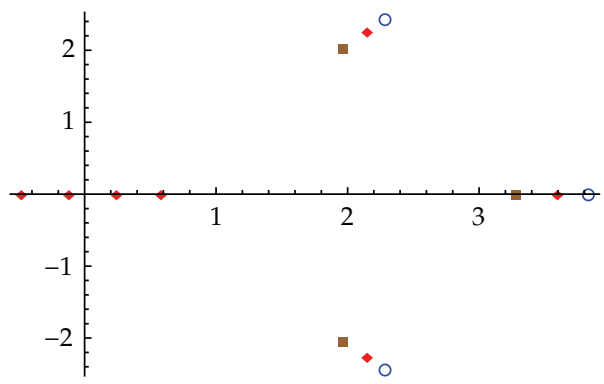

(c)

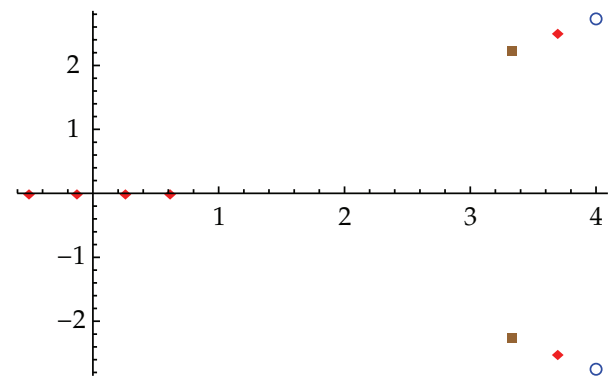

(b)

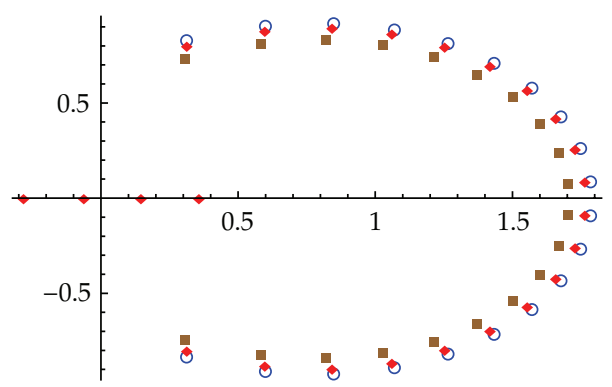

(d)

Figure 8: J2: distributions of the zeros $\bar{\eta}_{k}^{(\ell, n)}, \eta_{j}^{(\ell, n)}(\bullet), \bar{\xi}_{k}^{(\ell)}(\bigcirc)$, and $\xi_{k}^{(\ell)}(\mathbf{\square})$ for the J2 Jacobi polynomials, with $g=3, h=4$, and $n=4$. The four diagrams correspond to $\ell=1(\mathrm{a}), 2(\mathrm{~b}), 3(\mathrm{c})$, and 20(d), respectively.

Generally, for even $\ell$, there are $\ell$ complex zeros with

$$
\mathfrak{R} \bar{\xi}_{k}^{(\ell)}>\mathfrak{R} \xi_{k}^{(\ell)}, \quad\left|\Im \bar{\xi}_{k}^{(\ell)}\right|>\left|\Im \xi_{k}^{(\ell)}\right|, \quad k=1,2, \ldots, \frac{\ell}{2} .
$$

As $\ell$ changes to $\ell+1$ which is odd, all zeros move toward the $y$-axis, with the real parts decreased, and a new real zero appears to the right of all the complex zeros on the real $x$ axis. As $\ell$ increases further, the complex zeros move as described before, and the zero on the real axis bifurcates into two complex zeros, giving an even number of complex zeros. The absolute value of the imaginary part of the complex zeros may increase initially, but eventually decrease as $\ell$ increases. This pattern continues as $\ell$ increases.

Figures 8 and 9 show these behaviors for some selected parameters. For large $\ell$, these zeros distribute in a horse-shoe pattern.

\section{Ordinary Zeros of $X_{\ell}$ Polynomials}

In the case of $X_{\ell}$ polynomials $P_{\ell, n}(\eta ; \mathcal{l})$, it has $n$ zeros in the (ordinary) domain, where the weight function is defined, that is, $(0, \infty)$ for the L1 and L2 polynomials and $(-1,1)$ for the $\mathrm{J} 1$ and J2 polynomials. The behavior of these zeros is the same as that of other ordinary orthogonal polynomials. See, for example, [18] and/or [19, Section 5.4]. 


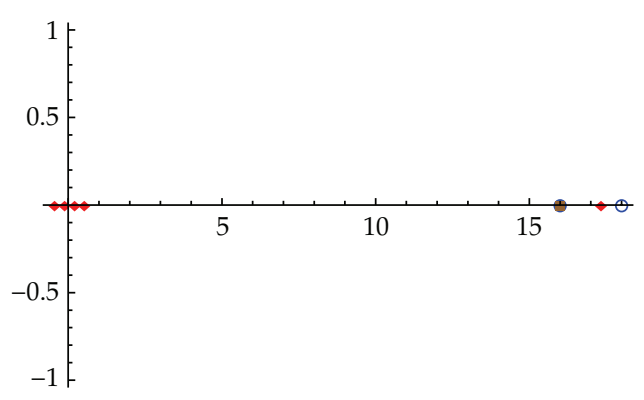

(a)

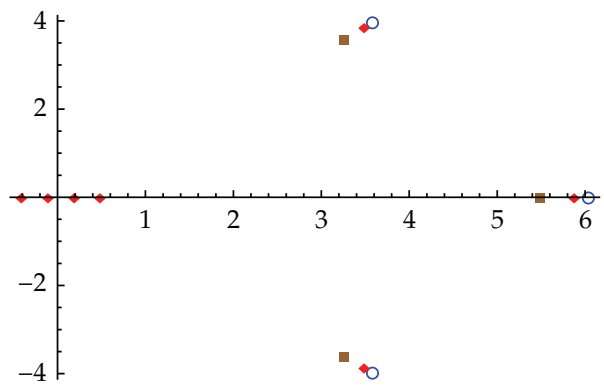

(c)

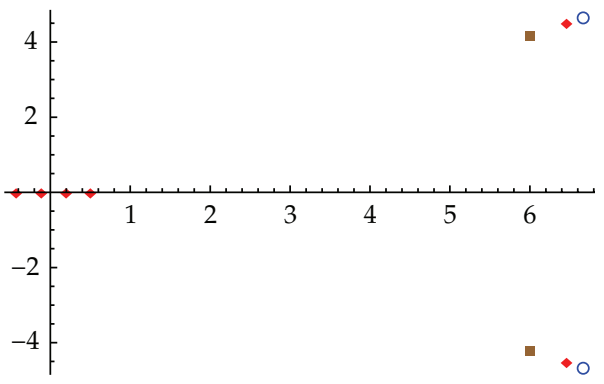

(b)

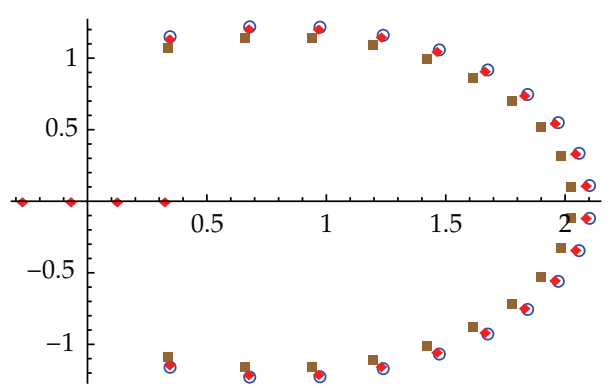

(d)

Figure 9: J2: distributions of the zeros $\bar{\eta}_{k}^{(\ell, n)}, \eta_{j}^{(\ell, n)}(\diamond), \bar{\xi}_{k}^{(\ell)}(\bigcirc)$, and $\xi_{k}^{(\ell)}(\boldsymbol{\square})$ for the J2 Jacobi polynomials, with $g=7, h=8$, and $n=4$. The four diagrams correspond to $\ell=1(\mathrm{a}), 2(\mathrm{~b}), 3(\mathrm{c})$, and 20(d), respectively.

\subsection{Behaviors as $n$ Increases at Fixed $l$}

This is guaranteed by the oscillation theorem of the one-dimensional quantum mechanics, since $P_{\ell, n}(\eta ; \lambda)$ are obtained as the polynomial parts of the eigenfunctions of a shape invariant quantum mechanical problem. Explicitly, as $n$ changes to $n+1$, all zeros of $P_{\ell, n}$ decrease, and a new zero appears from the right. Thus the $n$ zeros of $P_{\ell, n}(\eta ; \lambda)$ and the $n+1$ zeros of $P_{\ell, n+1}(\eta ; \mathcal{l})$ interlace with each other: each zero of $P_{\ell, n}(\eta ; \mathcal{l})$ is surrounded by two zeros of $P_{\ell, n+1}(\eta ; \lambda)$.

Figures 1-3 show these behaviors for selected parameters.

\subsection{Behaviors as $\ell$ Increases at Fixed $n$}

From Figures 4-7, one sees that for L1 and L2 Laguerre polynomials (whose zeros are positive in the ordinary domains), all the $n$ zeros shift to the right as $\ell$ increases.

For J2 Jacobi polynomials, the positive (negative) zeros shift left (right) as $\ell$ increases, that is, they move toward the origin $\eta=0$. This is illustrated in Figures 8 and 9. 


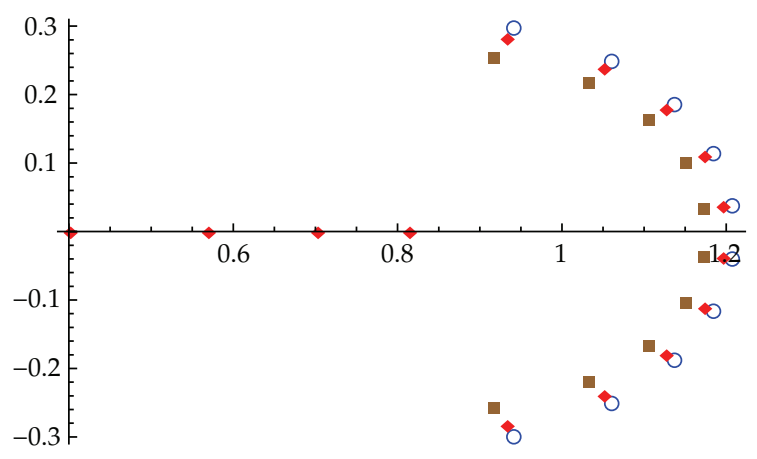

(a)

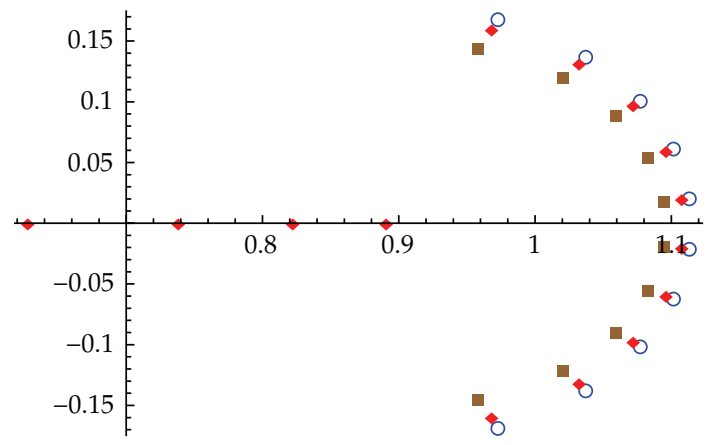

(b)

Figure 10: J2: distributions of the zeros $\bar{\eta}_{k}^{(\ell, n)}, \eta_{j}^{(\ell, n)}(\downarrow), \bar{\xi}_{k}^{(\ell)}(\bigcirc)$, and $\xi_{k}^{(\ell)}(\square)$ for the J2 Jacobi polynomials, with $g=2, \ell=10$, and $n=4$. The two diagrams correspond to $h=50$ (a) and 100(b), respectively.

\subsection{Additional Observation for the L1 Case}

Using the well-known derivative relation

$$
\partial_{\eta} L_{n}^{(\alpha)}(\eta)=-L_{n-1}^{(\alpha+1)}(\eta)
$$

and

$$
L_{n}^{(\alpha)}(\eta)-L_{n}^{(\alpha-1)}(\eta)=L_{n-1}^{(\alpha)}(\eta)
$$

we get

$$
\begin{aligned}
P_{\ell, \ell}(\eta ; g)= & L_{\ell}^{(g+\ell-(1 / 2))}(-\eta) L_{\ell}^{(g+\ell-(3 / 2))}(\eta)+L_{\ell}^{(g+\ell-(1 / 2))}(\eta) L_{\ell}^{(g+\ell-(3 / 2))}(-\eta) \\
& -L_{\ell}^{(g+\ell-(3 / 2))}(\eta) L_{\ell}^{(g+\ell-(3 / 2))}(-\eta) .
\end{aligned}
$$

Hence, when $n=\ell$, the L1 Laguerre is an even function of $\eta$, and its zeros are symmetric with respect to $\eta=0$. 
Table 1: List of the zeros $\bar{\xi}_{k}^{(\ell)}, \xi_{k}^{(\ell)}$, and $\bar{\eta}_{k}^{(\ell, n)}$ for the L1 Laguerre polynomials with $g=2, \ell=5$, and $n=0,10$, $20, \ldots, 60(k=1,2, \ldots, \ell)$. It can be seen that when $n=0, \bar{\eta}_{k}^{(\ell, n=0)}=\bar{\xi}_{k}^{(\ell)}$. As $n$ increases, $\bar{\eta}_{k}^{(\ell, n)}$ approaches to $\xi_{k}^{(\ell)}$.

\begin{tabular}{lcccccc}
\hline $\bar{\xi}_{k}^{(\ell)}:$ & & -22.4802 & -15.2391 & -10.1403 & -6.2977 & -3.3427 \\
\hline & $n=0$ & -22.4802 & -15.2391 & -10.1403 & -6.2977 & -3.3427 \\
& 10 & -22.0686 & -14.8767 & -9.8314 & -6.0505 & -3.1698 \\
& 20 & -21.8830 & -14.7189 & -9.7004 & -5.9469 & -3.0962 \\
$\bar{\eta}_{k}^{(\ell, n)}:$ & 30 & -21.7717 & -14.6253 & -9.6233 & -5.8862 & -3.0529 \\
& 40 & -21.6954 & -14.5617 & -9.5711 & -5.8452 & -3.0237 \\
& 50 & -21.6390 & -14.5148 & -9.5327 & -5.8152 & -3.0022 \\
& 60 & -21.5951 & -14.4784 & -9.5030 & -5.7919 & -2.9856 \\
\hline$\xi_{k}^{(\ell)}:$ & & -21.0456 & -14.0274 & -9.1375 & -5.5071 & -2.7824 \\
\hline
\end{tabular}

Table 2: Same as Table 1 for L1 Laguerre polynomials with $g=8$ and $\ell=5$.

\begin{tabular}{lcccccc}
\hline $\bar{\xi}_{k}^{(\ell)}:$ & & -30.7592 & -22.3415 & -16.1499 & -11.2032 & -7.0462 \\
\hline & $n=0$ & -30.7592 & -22.3415 & -16.1499 & -11.2032 & -7.0462 \\
& 10 & -30.4724 & -22.0859 & -15.9269 & -11.0165 & -6.9029 \\
& 20 & -30.3144 & -21.9474 & -15.8074 & -10.9169 & -6.8255 \\
$\bar{\eta}_{k}^{(\ell, n)}:$ & 30 & -30.2107 & -21.8574 & -15.7301 & -10.8525 & -6.7752 \\
& 40 & -30.1361 & -21.7928 & -15.6748 & -10.8065 & -6.7393 \\
& 50 & -30.0791 & -21.7436 & -15.6328 & -10.7715 & -6.7119 \\
& 60 & -30.0336 & -21.7046 & -15.5994 & -10.7438 & -6.6902 \\
\hline$\xi_{k}^{(\ell)}:$ & & -29.4106 & -21.1735 & -15.1488 & -10.3703 & -6.3968 \\
\hline
\end{tabular}

Table 3: Same as Table 1 but for L2 Laguerre polynomials with $g=3$ and $\ell=4$.

\begin{tabular}{lccc}
\hline $\bar{\xi}_{k}^{(\ell)}:$ & $-5.29007 \pm 1.65310 i$ & $-3.70993 \pm 5.05130 i$ \\
\hline & $n=0$ & $-5.29007 \pm 1.65310 i$ & $-3.70993 \pm 5.05130 i$ \\
& 10 & $-4.84198 \pm 1.57129 i$ & $-3.25524 \pm 4.78004 i$ \\
$\bar{\eta}_{k}^{(\ell, n)}:$ & 20 & $-4.71299 \pm 1.54888 i$ & $-3.12839 \pm 4.70776 i$ \\
& 30 & $-4.64523 \pm 1.53732 i$ & $-3.06246 \pm 4.67065 i$ \\
& 40 & $-4.60183 \pm 1.52998 i$ & $-3.02046 \pm 4.64713 i$ \\
& 50 & $-4.57100 \pm 1.52479 i$ & $-2.99074 \pm 4.63053 i$ \\
$\xi_{k}^{(\ell)}:$ & 60 & $-4.54766 \pm 1.52087 i$ & $-2.96828 \pm 4.61801 i$ \\
\hline
\end{tabular}

Table 4: Same as Table 3 for L2 Laguerre polynomials with $g=10$ and $\ell=5$.

\begin{tabular}{lcccc}
\hline $\bar{\xi}_{k}^{(\ell)}:$ & -12.8111 & $-12.2115 \pm 4.7185 i$ & $-10.1329 \pm 9.7965 i$ \\
\hline & $n=0$ & -12.8111 & $-12.2115 \pm 4.7185 i$ & $-10.1329 \pm 9.7965 i$ \\
& 10 & -12.5476 & $-11.9465 \pm 4.6639 i$ & $-9.8622 \pm 9.6780 i$ \\
$\bar{\eta}_{k}^{(\ell, n)}:$ & 20 & -12.4210 & $-11.8198 \pm 4.6384 i$ & $-9.7348 \pm 9.6233 i$ \\
& 30 & -12.3430 & $-11.7418 \pm 4.6229 i$ & $-9.6570 \pm 9.5901 i$ \\
& 40 & -12.2888 & $-11.6877 \pm 4.6122 i$ & $-9.6032 \pm 9.5673 i$ \\
& 50 & -12.2483 & $-11.6473 \pm 4.6043 i$ & $-9.5632 \pm 9.5504 i$ \\
$\bar{\xi}_{k}^{(\ell)}:$ & 60 & -12.2165 & $-11.6157 \pm 4.5981 i$ & $-9.5319 \pm 9.5372 i$ \\
\hline
\end{tabular}


Table 5: Same as Table 1 but for J2 Jacobi polynomials with $g=3, h=4$, and $\ell=4$.

\begin{tabular}{lccc}
\hline $\bar{\xi}_{k}^{(\ell)}:$ & & $1.56846 \pm 2.10278 i$ & $3.00297 \pm 0.91199 i$ \\
\hline & $n=0$ & $1.56846 \pm 2.10278 i$ & $3.00297 \pm 0.91199 i$ \\
& 10 & $1.45201 \pm 1.89890 i$ & $2.76834 \pm 0.82626 i$ \\
& 20 & $1.42407 \pm 1.85433 i$ & $2.71360 \pm 0.80733 i$ \\
$\bar{\eta}_{k}^{(\ell, n)}:$ & 30 & $1.41139 \pm 1.83435 i$ & $2.68882 \pm 0.79884 i$ \\
& 40 & $1.40414 \pm 1.82297 i$ & $2.67466 \pm 0.79401 i$ \\
& 50 & $1.39944 \pm 1.81561 i$ & $2.66550 \pm 0.79088 i$ \\
& 60 & $1.39615 \pm 1.81046 i$ & $2.65907 \pm 0.78869 i$ \\
\hline$\xi_{k}^{(\ell)}:$ & & $1.37745 \pm 1.78118 i$ & $2.62255 \pm 0.77624 i$ \\
\hline
\end{tabular}

Table 6: Same as Table 5 for J2 Jacobi polynomials with $g=3, h=4$, and $\ell=5$.

\begin{tabular}{lclll}
\hline $\bar{\xi}_{k}^{(\ell)}:$ & $1.19188 \pm 1.85256 i$ & $2.38851 \pm 1.21416 i$ & 2.83923 \\
\hline & $n=0$ & $1.19188 \pm 1.85256 i$ & $2.38851 \pm 1.21416 i$ & 2.83923 \\
& 10 & $1.11856 \pm 1.68660 i$ & $2.22979 \pm 1.11021 i$ & 2.64753 \\
& 20 & $1.09936 \pm 1.64851 i$ & $2.18998 \pm 1.08600 i$ & 2.59983 \\
$\bar{\eta}_{k}^{(\ell, n)}:$ & 30 & $1.09041 \pm 1.63110 i$ & $2.17151 \pm 1.07491 i$ & 2.57771 \\
& 40 & $1.08522 \pm 1.62106 i$ & $2.16081 \pm 1.06852 i$ & 2.56490 \\
& 50 & $1.08184 \pm 1.61453 i$ & $2.15382 \pm 1.06436 i$ & 2.55654 \\
& 60 & $1.07945 \pm 1.60993 i$ & $2.14890 \pm 1.06143 i$ & 2.55066 \\
\hline$\xi_{k}^{(\ell)}:$ & $1.06566 \pm 1.58339 i$ & $2.12047 \pm 1.04452 i$ & 2.51663 \\
\hline
\end{tabular}

\section{Asymptotic Behavior of $\bar{\eta}_{k}^{(\ell, n)} \rightarrow \xi_{k}^{(\ell)}$ as $n \rightarrow \infty$}

Here we provide intuitive arguments for the above asymptotic behavior. As mentioned before, for $n=0$, we have $\bar{\eta}_{k}^{(\ell, 0)}=\bar{\xi}_{k}^{(\ell)}$, as $P_{n=0}=1$. We will show that as $n \rightarrow \infty, \bar{\eta}_{k}^{(\ell, n)} \rightarrow \xi_{k}^{(\ell)}$. This amounts to showing that in this limit, $\partial_{\eta} P_{n}$ dominates over $P_{n}$.

\subsection{L1 and L2 Cases}

We will make use of the above derivative relation (4.1) and (Perron) Theorem 8.22.3 of [17], namely,

$$
L_{n}^{(\alpha)}(\eta) \cong \frac{e^{\eta / 2}}{2 \sqrt{\pi}}(-\eta)^{-((\alpha / 2)-(1 / 4))} n^{((\alpha / 2)-(1 / 4))} e^{2 \sqrt{-n \eta}}, \quad \alpha \in \mathbb{R}, \eta \in \mathbb{C} \backslash(0, \infty),
$$

which gives the asymptotic form of $L_{n}^{(\alpha)}(\eta)$ for large $n$. For the L1 and L2 cases, we have $\alpha=g+\ell-3 / 2$ and $g+\ell+1 / 2$, respectively.

One finds

$$
\left|\frac{L_{n}^{(\alpha)}(\eta)}{\partial_{\eta} L_{n}^{(\alpha)}(\eta)}\right| \sim\left|-\frac{1}{\sqrt{n}}(-\eta)^{1 / 2}\right| .
$$


Table 7: Same as Table 5 for J2 Jacobi polynomials with $g=8, h=9$, and $\ell=3$.

\begin{tabular}{lccc}
\hline $\bar{\xi}_{k}^{(\ell)}:$ & & $3.90615 \pm 4.35051 i$ & 6.58770 \\
\hline & $n=0$ & $3.90615 \pm 4.35051 i$ & 6.58770 \\
& 10 & $3.74981 \pm 4.16635 i$ & 6.32188 \\
& 20 & $3.69527 \pm 4.10323 i$ & 6.22948 \\
$\bar{\eta}_{k}^{(\ell, n)}:$ & 30 & $3.66745 \pm 4.07116 i$ & 6.18240 \\
& 40 & $3.65057 \pm 4.05174 i$ & 6.15383 \\
& 50 & $3.63924 \pm 4.03870 i$ & 6.13465 \\
& 60 & $3.63110 \pm 4.02935 i$ & 6.12088 \\
\hline$\xi_{k}^{(\ell)}:$ & & $3.58151 \pm 3.97238 i$ & 6.03699 \\
\hline
\end{tabular}

For large $n$ with fixed $\eta, \partial_{\eta} L_{n}^{(\alpha)}(\eta)$ dominates over $L_{n}^{(\alpha)}(\eta)$, and thus the zeros of $P_{\ell, n}$ are determined by those of $\xi_{\ell}(\eta ; g)$ as $n \rightarrow \infty$.

\subsection{J2 Jacobi}

For the asymptotic form of $P_{n}^{(\alpha, \beta)}(\eta)$ for large $n$, we will make use of Theorem 8.21.7 of [17]:

$$
\begin{aligned}
P_{n}^{(\alpha, \beta)}(\eta) \cong & (\eta-1)^{-\alpha / 2}(\eta+1)^{-\beta / 2}\{\sqrt{\eta+1}+\sqrt{\eta-1}\}^{\alpha+\beta} \\
& \times \frac{\left(\eta^{2}-1\right)^{-1 / 4}}{\sqrt{2 \pi n}}\left\{\eta+\sqrt{\eta^{2}-1}\right\}^{n+(1 / 2)}, \quad \alpha, \beta \in \mathbb{R}, \eta \in \mathbb{C} \backslash[-1,1],
\end{aligned}
$$

and

$$
\partial_{\eta} P_{n}^{(\alpha, \beta)}(\eta)=\frac{1}{2}(n+\alpha+\beta+1) P_{n-1}^{(\alpha+1, \beta+1)}(\eta)
$$

One finds

$$
\frac{P_{n}^{(\alpha, \beta)}(\eta)}{\partial_{\eta} P_{n}^{(\alpha, \beta)}(\eta)} \sim \frac{2}{(n+\alpha+\beta+1)} \sqrt{\frac{n-1}{n}}\left(\eta^{2}-1\right)^{1 / 2} \frac{\eta+\sqrt{\eta^{2}-1}}{(\sqrt{\eta+1}+\sqrt{\eta-1})^{2}} .
$$

Again, for large $n$ with fixed $\eta, \partial_{\eta} P_{n}^{(\alpha, \beta)}(\eta)$ dominates over $P_{n}^{(\alpha, \beta)}(\eta)$, and thus the zeros of $P_{\ell, n}$ are determined by those of $\xi_{\ell}(\eta ; g)$ as $n \rightarrow \infty$. 


\section{Behaviors at Large $g$ and/or $h$}

\subsection{L1 Laguerre}

As $g$ increases, we have that

$$
\left|\bar{\xi}_{k}^{(\ell)}\right|, \quad\left|\xi_{k}^{(\ell)}\right|, \quad\left|\bar{\eta}_{k}^{(\ell, n)}\right|, \quad\left|\eta_{k}^{(\ell, n)}\right|
$$

all increase. That is, all the zeros move away from the $y$-axis. This can be seen from Figures 4 and 5.

In fact, for large $g$, we have $\xi_{\ell}(\eta ; g+1) \approx \xi_{\ell}(\eta ; g)$. Hence

$$
\begin{aligned}
P_{\ell, n}(\eta ; g) & \approx \xi_{\ell}(\eta ; g)\left[L_{n}^{(g+\ell-(3 / 2))}(\eta)-\partial_{\eta} L_{n}^{(g+\ell-(3 / 2))}(\eta)\right] \\
& \approx \xi_{\ell}(\eta ; g) L_{n}^{(g+\ell-(1 / 2))}(\eta) .
\end{aligned}
$$

For $g \gg 1, P_{\ell, n}(\eta ; g)$ approaches

$$
P_{\ell, n}(\eta ; g) \approx L_{\ell}^{(g+\ell)}(-\eta) L_{n}^{(g+\ell)}(\eta)
$$

Thus the extra $\left(\bar{\eta}_{k}^{(\ell, n)}\right)$ and the ordinary $\left(\eta_{k}^{(\ell, n)}\right)$ zeros of $P_{\ell, n}(\eta ; g)$ are given by the zeros of $L_{\ell}^{(g+\ell)}(-\eta)$ and $L_{n}^{(g+\ell)}(\eta)$, respectively.

\subsection{L2 Laguerre}

As $g$ increases, we have $\mathfrak{R} \bar{\xi}_{k}^{(\ell)}, \mathfrak{R} \xi_{k}^{(\ell)}$ decreased, $\left|\Im \xi_{k}^{(\ell)}\right|,\left|\Im \xi_{k}^{(\ell)}\right|$ increased, and $\eta_{k}^{(\ell, n)}$ increased. This is easily seen from Figures 6 and 7. That is, the zeros $\bar{\xi}_{k}^{(\ell)}, \xi_{k}^{(\ell)}$, and hence $\bar{\eta}_{k}^{(\ell, n)}$ all are moving leftwards and away from the $x$-axis, while the ordinary zeros $\eta_{k}^{(\ell, n)}$ are moving towards the right.

In fact, for large $g$, we have $\xi_{\ell}(\eta ; g+1) \approx \xi_{\ell}(\eta ; g)$. Hence

$$
P_{\ell, n}(\eta ; g) \approx \xi_{\ell}(\eta ; g)\left[\left(g+\frac{1}{2}\right) L_{n}^{(g+\ell+(1 / 2))}(\eta)+\eta \partial_{\eta} L_{n}^{(g+\ell+(1 / 2))}(\eta)\right]
$$

Using (E.2), (E.10), and (E.9) of [9], we arrive at

$$
P_{\ell, n}(\eta ; g) \approx \xi_{\ell}(\eta ; g)\left[\left(g+\ell+\frac{1}{2}+n\right) L_{n}^{(g+\ell-(1 / 2))}(\eta)-\ell L_{n}^{(g+\ell+(1 / 2))}(\eta)\right]
$$

For $g \gg 1, P_{\ell, n}(\eta ; g)$ approaches

$$
P_{\ell, n}(\eta ; g) \approx L_{\ell}^{(-g-\ell)}(\eta) L_{n}^{(g+\ell)}(\eta)
$$


Thus the extra $\left(\bar{\eta}_{k}^{(\ell, n)}\right)$ and the ordinary $\left(\eta_{k}^{(\ell, n)}\right)$ zeros of $P_{\ell, n}(\eta ; g)$ are given by the zeros of $L_{\ell}^{(-g-\ell)}(\eta)$ and $L_{n}^{(g+\ell)}(\eta)$, respectively.

\subsection{J2 Jacobi}

As $g, h$ increases, we have $\mathfrak{R} \bar{\xi}_{k}^{(\ell)}, \mathfrak{R} \xi_{k}^{(\ell)},\left|\Im \bar{\xi}_{k}^{(\ell)}\right|,\left|\Im \xi_{k}^{(\ell)}\right|$ increased, as is evident from Figures 8 and 9. The extra zeros $\bar{\eta}_{k}^{(\ell, n)}$, being in between these zeros, follow the same pattern. That is, the zeros $\bar{\xi}_{k}^{(\ell)}, \xi_{k}^{(\ell)}$, and hence $\bar{\eta}_{k}^{(\ell, n)}$ all are moving away from the $x$ and $y$-axes. The ordinary zeros $\eta_{k}^{(\ell, n)}$ will have their norm $\left|\eta_{k}^{(\ell, n)}\right|$ decrease in general as $g$ increases. Thus these zeros move towards the $y$-axis.

In fact, for large $g$ and $h$, we have $(\alpha \equiv g+\ell+(1 / 2), \beta \equiv h+\ell-(3 / 2))$ as

$$
P_{\ell, n}(\eta ; g, h) \approx \xi_{\ell}(\eta ; g, h)\left[\left(g+\frac{1}{2}\right) P_{n}^{(\alpha, \beta)}(\eta)-(1-\eta) \partial_{\eta} P_{n}^{(\alpha, \beta)}(\eta)\right]
$$

Using (E.13) and (E.23) of [9], we arrive at

$$
P_{\ell, n}(\eta ; g, h) \approx \xi_{\ell}(\eta ; g, h)\left[(\alpha+n) P_{n}^{(\alpha+1, \beta+1)}(\eta)-\ell P_{n}^{(\alpha, \beta)}(\eta)\right]
$$

For $g \gg 1$ and $h \gg 1, P_{\ell, n}(\eta ; g, h)$ approaches

$$
P_{\ell, n}(\eta ; g) \approx P_{\ell}^{(-g-\ell, h+\ell)}(\eta) P_{n}^{(g+\ell, h+\ell)}(\eta)
$$

Thus the extra $\left(\bar{\eta}_{k}^{(\ell, n)}\right)$ and the ordinary $\left(\eta_{k}^{(\ell, n)}\right)$ zeros of $P_{\ell, n}(\eta ; g, h)$ are given by the zeros of $P_{\ell}^{(-g-\ell, h+\ell)}(\eta)$ and $P_{n}^{(g+\ell, h+\ell)}(\eta)$, respectively.

\subsubsection{Additional Observation: $h \gg g$}

For $h \gg g$, all zeros, that is, $\bar{\xi}_{j}^{(\ell)}, \xi_{j}^{(\ell)}, \bar{\eta}_{k}^{(\ell, n)}, \eta_{k}^{(\ell, n)}$, gather around $\eta=1$. This can be understood as follows. From the series expansion of the Jacobi polynomials, (A.2)

$$
P_{n}^{(\alpha, \beta)}(\eta)=\frac{(\alpha+1)_{n}}{n !} \sum_{k=0}^{n} \frac{1}{k !} \frac{(-n)_{k}(n+\alpha+\beta+1)_{k}}{(\alpha+1)_{k}}\left(\frac{1-\eta}{2}\right)^{k}
$$

one sees that, for $h \gg g$, the absolute value of $P_{\ell, n}(\eta ; g, h)$ is large near $\eta=-1$ and small at $\eta=1$. Hence, in this limit, the zeros of $P_{\ell, n}(\eta ; g, h)$ distribute very near $\eta=1$. We show this in Figure 10 for certain parameters. 


\section{Summary}

The discovery of new types of orthogonal polynomials, called the exceptional $X_{\ell}$ Laguerre and Jacobi polynomials, has aroused great interest in the last two years. Unlike the wellknown classical orthogonal polynomials which start with constant terms, these new polynomials $P_{\ell, n}(\eta ; \lambda)$ have the lowest degree $\ell=1,2, \ldots$, and yet they form a complete set with respect to some positive-definite measure. Many essential properties have been studied in [9].

In this paper, we have considered the distributions of the zeros of these new polynomials as some parameters of the Hamiltonians change based on numerical analysis. The $X_{\ell}$ polynomial $P_{\ell, n}(\eta ; \mathcal{l})$ has $n$ zeros in the ordinary domain where the weight function is defined, that is, $(0, \infty)$ for the L1 and L2 polynomials and $(-1,1)$ for the J1 and J2 polynomials. The behavior of these ordinary zeros are the same as those of other ordinary orthogonal polynomials. In addition to these $n$ zeros, there are extra $l$ zeros outside the ordinary domain.

For the ordinary zeros, their distribution as $n$ increases at a fixed $\ell$ follows the patterns of the zeros of the ordinary classical orthogonal polynomials: they are governed by the oscillation theorem, and the $n+1$ zeros of $P_{\ell, n+1}(\eta ; \mathcal{l})$ interlace with the $n$ zeros of $P_{\ell, n}(\eta ; \lambda)$. On the other hand, when $\ell$ increases at a fixed $n$, the type L1 and L2 Laguerre polynomials will have all their $n$ zeros shifted to the right. For the J1 and the J2 Jacobi polynomials, both the positive and negative zeros move toward the origin $\eta=0$ as $\ell$ increases.

For the $\ell$ extra zeros of $P_{\ell, n}(\eta ; \mathcal{l})$, each and everyone of them is sandwiched between the corresponding zeros of the deforming polynomials $\xi_{\ell}(\eta ; \boldsymbol{\lambda}+\boldsymbol{\delta})$ and $\xi_{\ell}(\eta ; \boldsymbol{\lambda})$. As $n$ increases at a fixed $\ell$, the extra zeros move from the zeros of $\xi_{\ell}(\eta ; \boldsymbol{\lambda}+\delta)$ to those of $\xi_{\ell}(\eta ; \lambda)$.

The behaviors of the extra zeros as $\ell$ increases at a fixed $n$ are more complex. For the L1 Laguerre polynomials, all the extra zeros lie on the negative $x$-axis. So as $\ell$ increases by one, the number of the extra zeros increases from $\ell$ to $\ell+1$. For the L2 Laguerre and J1 and J2 Jacobi polynomials, they have $\ell / 2$ pairs of complex zeros for even $\ell$, and $(\ell-1) / 2$ pairs of complex zeros and a real zero outside the ordinary domains where the weight functions are defined. As $\ell$ increases, all the complex zeros move toward the right in the case of the L2 Laguerre and J1 Jacobi polynomials, and toward the left for the J2 Jacobi polynomials, while the extra real zeros bifurcate into new pairs of complex zeros. For large $\ell$, these zeros appear to distribute symmetrically with respect to the $x$-axis in horse-shoe patterns. It is interesting to note that in the asymptotic regions of the parameters $(g \gg 1, h \gg 1)$, the exceptional polynomial $P_{\ell, n}(\eta, \lambda)$ is expressed as the product of the original polynomial $P_{n}(\eta)$ and the deforming polynomial $\xi_{\ell}(\eta ; \lambda),(6.3),(6.6)$, and (6.9). After completing this paper, we became aware of some new works on exceptional orthogonal polynomials [2123].

\section{Appendix}

\section{Explicit Forms of Some Lower Degree Exceptional Orthogonal Polynomials}

In the Appendix we provide, for self-containedness, the definitions of the classical Laguerre and Jacobi polynomials and the explicit forms of some lower degree members of the exceptional orthogonal polynomials. Here the independent variable is denoted by $x$. 
The Classical Laguerre Polynomials

The degree $n$ classical Laguerre polynomial is defined by

$$
L_{n}^{(\alpha)}(x)=\frac{1}{n !} \sum_{k=0}^{n} \frac{(-n)_{k}}{k !}(\alpha+k+1)_{n-k} x^{k}
$$

The Classical Jacobi Polynomials

The degree $n$ classical Jacobi polynomial is defined by

$$
P_{n}^{(\alpha, \beta)}(x)=\frac{(\alpha+1)_{n}}{n !} \sum_{k=0}^{n} \frac{1}{k !} \frac{(-n)_{k}(n+\alpha+\beta+1)_{k}}{(\alpha+1)_{k}}\left(\frac{1-x}{2}\right)^{k}
$$

In these formulas, $(a)_{n} \stackrel{\text { def }}{=} \prod_{k=0}^{n-1}(a+k)$ is the shifted factorial (the Pochhammer symbol).

\section{L1 Exceptional Laguerre Polynomials}

$$
\begin{gathered}
P_{1,0}^{L 1}(x ; g)=\frac{1}{2}(3+2 g)+x, \\
P_{1,1}^{L 1}(x ; g)=\frac{1}{4}(1+2 g)(5+2 g)-x^{2}, \\
P_{1,2}^{L 1}(x ; g)=\frac{1}{16}(1+2 g)(3+2 g)(7+2 g)-\frac{1}{8}(1+2 g)(7+2 g) x-\frac{1}{4}(7+2 g) x^{2}+\frac{1}{2} x^{3}, \\
P_{2,0}^{L 1}(x ; g)=\frac{1}{8}(5+2 g)(7+2 g)+\frac{1}{2}(7+2 g) x+\frac{1}{2} x^{2}, \\
P_{2,1}^{L 1}(x ; g)=\frac{1}{16}(3+2 g)(5+2 g)(9+2 g)+\frac{1}{8}(3+2 g)(9+2 g) x-\frac{1}{4}(9+2 g) x^{2}-\frac{1}{2} x^{3}, \\
P_{2,2}^{L 1}(x ; g)=\frac{1}{64}(3+2 g)(5+2 g)^{2}(11+2 g)-\frac{1}{8}(5+2 g)(11+2 g) x^{2}+\frac{1}{4} x^{4}, \\
P_{3,0}^{L 1}(x ; g)=\frac{1}{48}(7+2 g)(9+2 g)(11+2 g)+\frac{1}{8}(9+2 g)(11+2 g) x+\frac{1}{4}(11+2 g) x^{2}+\frac{1}{6} x^{3}, \\
P_{3,1}^{L 1}(x ; g)=\frac{1}{96}(5+2 g)(7+2 g)(9+2 g)(13+2 g)+\frac{1}{24}(5+2 g)(9+2 g)(13+2 g) x \\
-\frac{1}{4}(13+2 g) x^{2}-\frac{1}{6}(13+2 g) x^{3}-\frac{1}{6} x^{4} .
\end{gathered}
$$


L2 Exceptional Laguerre Polynomials

$$
\begin{gathered}
P_{1,0}^{L 2}(x ; g)=-\frac{1}{2}(3+2 g)-x, \\
P_{1,1}^{L 2}(x ; g)=\frac{1}{4}(1+2 g)(5+2 g)-x^{2}, \\
P_{1,2}^{L 2}(x ; g)=-\frac{1}{16}(1+2 g)(3+2 g)(7+2 g)+\frac{1}{8}(1+2 g)(7+2 g) x+\frac{1}{4}(7+2 g) x^{2}-\frac{1}{2} x^{3}, \\
P_{2,0}^{L 2}(x ; g)=\frac{1}{8}(3+2 g)(5+2 g)+\frac{1}{2}(3+2 g) x+\frac{1}{2} x^{2}, \\
P_{2,1}^{L 2}(x ; g)=\frac{1}{16}(1+2 g)(5+2 g)(7+2 g)+\frac{1}{8}(1+2 g)(7+2 g) x-\frac{1}{4}(1+2 g) x^{2}-\frac{1}{2} x^{3}, \\
P_{2,2}^{L 2}(x ; g)=\frac{1}{64}(1+2 g)(3+2 g)(7+2 g)(9+2 g)-\frac{1}{8}(1+2 g)(9+2 g) x^{2}-x^{3}+\frac{1}{4} x^{4}, \\
P_{3,0}^{L 2}(x ; g)=-\frac{1}{48}(3+2 g)(5+2 g)(7+2 g)-\frac{1}{8}(3+2 g)(5+2 g) x-\frac{1}{4}(3+2 g) x^{2}-\frac{1}{6} x^{3}, \\
P_{3,1}^{L 2}(x ; g)=-\frac{1}{96}(1+2 g)(5+2 g)(7+2 g)(9+2 g)-\frac{1}{24}(1+2 g)(5+2 g)(9+2 g) x \\
-\frac{1}{4}(1+2 g) x^{2}+\frac{1}{6}(1+2 g) x^{3}+\frac{1}{6} x^{4} .
\end{gathered}
$$

J1 Exceptional Jacobi Polynomials

$$
\begin{aligned}
P_{1,0}^{J 1}(x ; g, h)=\frac{1}{2}(3+g+h)+\frac{1}{2}(g-h) x, & \\
P_{1,1}^{J 1}(x ; g, h)= & \frac{1}{4}(g-h)(3+g+h)+\frac{1}{4}\left(5+6 g+2 g^{2}+6 h+2 h^{2}\right) x \\
& +\frac{1}{4}(g-h)(3+g+h) x^{2}, \\
P_{1,2}^{J 1}(x ; g, h)= & \frac{1}{16}\left(-7-8 g+2 g^{2}+g^{3}-8 h-8 g h-g^{2} h+2 h^{2}-g h^{2}+h^{3}\right) \\
+ & \frac{1}{16}(g-h)\left(11+13 g+3 g^{2}+13 h+2 g h+3 h^{2}\right) x \\
+ & \frac{1}{16}(4+g+h)\left(7+8 g+3 g^{2}+8 h-2 g h+3 h^{2}\right) x^{2} \\
+ & \frac{1}{16}(g-h)(4+g+h)(5+g+h) x^{3},
\end{aligned}
$$




$$
\begin{aligned}
& P_{2,0}^{J 1}(x ; g, h)=\frac{1}{8}\left(23+9 g+g^{2}+11 h+2 g h+h^{2}\right)-\frac{1}{4}(-1-g+h)(5+g+h) x \\
& +\frac{1}{8}(-2-g+h)(-1-g+h) x^{2} \\
& P_{2,1}^{J 1}(x ; g, h)=\frac{1}{16}\left(8+23 g+9 g^{2}+g^{3}-23 h+2 g h+g^{2} h-11 h^{2}-g h^{2}-h^{3}\right) \\
& +\frac{1}{16}(5+g+h)\left(15+11 g+3 g^{2}+9 h-2 g h+3 h^{2}\right) x \\
& -\frac{1}{16}(-1-g+h)\left(42+22 g+3 g^{2}+18 h+2 g h+3 h^{2}\right) x^{2} \\
& +\frac{1}{16}(-2-g+h)(-1-g+h)(5+g+h) x^{3} \\
& P_{2,2}^{J 1}(x ; g, h)=\frac{1}{64}\left(-81-54 g+7 g^{2}+8 g^{3}+g^{4}-100 h-80 g h-10 g^{2} h+5 h^{2}\right. \\
& \left.-16 g h^{2}-2 g^{2} h^{2}+10 h^{3}+h^{4}\right) \\
& -\frac{1}{32}\left(3-67 g-61 g^{2}-20 g^{3}-2 g^{4}+75 h+65 h^{2}+20 h^{3}+2 h^{4}\right) x \\
& +\frac{1}{32}\left(252+291 g+153 g^{2}+36 g^{3}+3 g^{4}+250 h+80 g h+10 g^{2} h\right. \\
& \left.+125 h^{2}+16 g h^{2}+2 g^{2} h^{2}+30 h^{3}+3 h^{4}\right) x^{2} \\
& -\frac{1}{32}(-1-g+h)(6+g+h)\left(27+14 g+2 g^{2}+10 h+2 h^{2}\right) x^{3} \\
& +\frac{1}{64}(-2-g+h)(-1-g+h)(6+g+h)(7+g+h) x^{4} \\
& P_{3,0}^{J 1}(x ; g, h)=\frac{1}{48}(7+g+h)\left(39+11 g+g^{2}+17 h+2 g h+h^{2}\right) \\
& -\frac{1}{16}(-2-g+h)\left(45+13 g+g^{2}+15 h+2 g h+h^{2}\right) x \\
& +\frac{1}{16}(-3-g+h)(-2-g+h)(7+g+h) x^{2} \\
& -\frac{1}{48}(-4-g+h)(-3-g+h)(-2-g+h) x^{3}, \\
& P_{3,1}^{J 1}(x ; g, h)=-\frac{1}{96}(7+g+h)\left(-24-39 g-11 g^{2}-g^{3}+39 h-6 g h\right. \\
& \left.-g^{2} h+17 h^{2}+g h^{2}+h^{3}\right)
\end{aligned}
$$




$$
\begin{aligned}
& +\frac{1}{96}\left(1395+1223 g+443 g^{2}+70 g^{3}+4 g^{4}+905 h+286 g h+54 g^{2} h\right. \\
& \left.+4 g^{3} h+359 h^{2}+30 g h^{2}+70 h^{3}+4 g h^{3}+4 h^{4}\right) x \\
& -\frac{1}{32}(-2-g+h)(7+g+h)\left(37+16 g+2 g^{2}+12 h+2 h^{2}\right) x^{2} \\
& +\frac{1}{96}(-3-g+h)(-2-g+h)\left(135+46 g+4 g^{2}+38 h+4 g h+4 h^{2}\right) x^{3} \\
& -\frac{1}{96}(-4-g+h)(-3-g+h)(-2-g+h)(7+g+h) x^{4} .
\end{aligned}
$$

J2 Exceptional Jacobi Polynomials

$$
\begin{aligned}
& P_{1,0}^{J 2}(x ; g, h)=-\frac{1}{2}(3+g+h)-\frac{1}{2}(g-h) x, \\
& P_{1,1}^{J 2}(x ; g, h)=-\frac{1}{4}(g-h)(3+g+h)-\frac{1}{4}\left(5+6 g+2 g^{2}+6 h+2 h^{2}\right) x \\
& -\frac{1}{4}(g-h)(3+g+h) x^{2} \\
& P_{1,2}^{J 2}(x ; g, h)=\frac{1}{16}\left(7+8 g-2 g^{2}-g^{3}+8 h+8 g h+g^{2} h-2 h^{2}+g h^{2}-h^{3}\right) \\
& -\frac{1}{16}(g-h)\left(11+13 g+3 g^{2}+13 h+2 g h+3 h^{2}\right) x \\
& -\frac{1}{16}(4+g+h)\left(7+8 g+3 g^{2}+8 h-2 g h+3 h^{2}\right) x^{2} \\
& -\frac{1}{16}(g-h)(4+g+h)(5+g+h) x^{3}, \\
& P_{2,0}^{J 2}(x ; g, h)=\frac{1}{8}\left(23+11 g+g^{2}+9 h+2 g h+h^{2}\right)+\frac{1}{4}(-1+g-h)(5+g+h) x \\
& +\frac{1}{8}(-2+g-h)(-1+g-h) x^{2}, \\
& P_{2,1}^{J 2}(x ; g, h)=\frac{1}{16}\left(-8+23 g+11 g^{2}+g^{3}-23 h-2 g h+g^{2} h-9 h^{2}-g h^{2}-h^{3}\right) \\
& +\frac{1}{16}(5+g+h)\left(15+9 g+3 g^{2}+11 h-2 g h+3 h^{2}\right) x \\
& +\frac{1}{16}(-1+g-h)\left(42+18 g+3 g^{2}+22 h+2 g h+3 h^{2}\right) x^{2} \\
& +\frac{1}{16}(-2+g-h)(-1+g-h)(5+g+h) x^{3},
\end{aligned}
$$




$$
\begin{aligned}
& P_{2,2}^{J 2}(x ; g, h)=\frac{1}{64}\left(-81-100 g+5 g^{2}+10 g^{3}+g^{4}-54 h-80 g h-16 g^{2} h+7 h^{2}-10 g h^{2}\right. \\
& \left.-2 g^{2} h^{2}+8 h^{3}+h^{4}\right) \\
& +\frac{1}{32}\left(3+75 g+65 g^{2}+20 g^{3}+2 g^{4}-67 h-61 h^{2}-20 h^{3}-2 h^{4}\right) x \\
& +\frac{1}{32}\left(252+250 g+125 g^{2}+30 g^{3}+3 g^{4}+291 h+80 g h+16 g^{2} h\right. \\
& \left.+153 h^{2}+10 g h^{2}+2 g^{2} h^{2}+36 h^{3}+3 h^{4}\right) x^{2} \\
& +\frac{1}{32}(-1+g-h)(6+g+h)\left(27+10 g+2 g^{2}+14 h+2 h^{2}\right) x^{3} \\
& +\frac{1}{64}(-2+g-h)(-1+g-h)(6+g+h)(7+g+h) x^{4}, \\
& P_{3,0}^{J 2}(x ; g, h)=-\frac{1}{48}(7+g+h)\left(39+17 g+g^{2}+11 h+2 g h+h^{2}\right) \\
& -\frac{1}{16}(-2+g-h)\left(45+15 g+g^{2}+13 h+2 g h+h^{2}\right) x \\
& -\frac{1}{16}(-3+g-h)(-2+g-h)(7+g+h) x^{2} \\
& -\frac{1}{48}(-4+g-h)(-3+g-h)(-2+g-h) x^{3}, \\
& P_{3,1}^{J 2}(x ; g, h)=-\frac{1}{96}(7+g+h)\left(-24+39 g+17 g^{2}+g^{3}-39 h-6 g h+g^{2} h\right. \\
& \left.-11 h^{2}-g h^{2}-h^{3}\right) \\
& -\frac{1}{96}\left(1395+905 g+359 g^{2}+70 g^{3}+4 g^{4}+1223 h+286 g h+30 g^{2} h\right. \\
& \left.+4 g^{3} h+443 h^{2}+54 g h^{2}+70 h^{3}+4 g h^{3}+4 h^{4}\right) x \\
& -\frac{1}{32}(-2+g-h)(7+g+h)\left(37+12 g+2 g^{2}+16 h+2 h^{2}\right) x^{2} \\
& -\frac{1}{96}(-3+g-h)(-2+g-h)\left(135+38 g+4 g^{2}+46 h+4 g h+4 h^{2}\right) x^{3} \\
& -\frac{1}{96}(-4+g-h)(-3+g-h)(-2+g-h)(7+g+h) x^{4} \text {. }
\end{aligned}
$$

\section{Acknowledgments}

This work is supported in part by the National Science Council (NSC) of the Republic of China under Grant no. NSC NSC-99-2112-M-032-002-MY3 (C. Ho) and in part by Grantsin-Aid for Scientific Research from the Ministry of Education, Culture, Sports, Science and Technology, no. 19540179 (R. Sasaki). R. Sasaki wishes to thank the R.O.C.'s National Center 
for Theoretical Sciences and National Taiwan University for the hospitality extended to him during his visit in which part of the work was done.

\section{References}

[1] D. Gómez-Ullate, N. Kamran, and R. Milson, "An extended class of orthogonal polynomials defined by a Sturm-Liouville problem," Journal of Mathematical Analysis and Applications, vol. 359, no. 1, pp. 352-367, 2009.

[2] D. Gómez-Ullate David, N. Kamran, and R. Milson, "An extension of Bochner's problem: exceptional invariant subspaces," Journal of Approximation Theory, vol. 162, no. 5, pp. 987-1006, 2010.

[3] S. Odake and R. Sasaki, "Infinitely many shape invariant potentials and new orthogonal polynomials," Physics Letters B, vol. 679, no. 4, pp. 414-417, 2009.

[4] S. Odake and R. Sasaki, "Another set of infinitely many exceptional $\left(X_{\ell}\right)$ Laguerre polynomials," Physics Letters B, vol. 684, no. 2-3, pp. 173-176, 2009.

[5] S. Odake and R. Sasaki, "Infinitely many shape-invariant potentials and cubic identities of the Laguerre and Jacobi polynomials," Journal of Mathematical Physics, vol. 51, no. 5, Article ID 053513, 9 pages, 2010.

[6] C. Quesne, "Exceptional orthogonal polynomials, exactly solvable potentials and supersymmetry," Journal of Physics A, vol. 41, no. 39, Article ID 392001, 6 pages, 2008.

[7] C. Quesne, "Solvable rational potentials and exceptional orthogonal polynomials in supersymmetric quantum mechanics," SIGMA, vol. 5, article 084, 24 pages, 2009.

[8] B. Bagchi, C. Quesne, and R. Roychoudhury, "Isospectrality of conventional and new extended potentials, second-order supersymmetry and role of PT symmetry," Pramana-Journal of Physics, vol. 73, no. 2, pp. 337-347, 2009.

[9] C. L. Ho, S. Odake, and R. Sasaki, "Properties of the exceptional $\left(X_{\ell}\right)$ Laguerre and Jacobi polynomials," SIGMA, vol. 7, article 107, 24 pages, 2011.

[10] D. Dutta and P. Roy, "Conditionally exactly solvable potentials and exceptional orthogonal polynomials," Journal of Mathematical Physics, vol. 51, no. 4, Article ID 042101, 9 pages, 2010.

[11] S. Bochner, "Über Sturm-Liouvillesche Polynomsysteme," Mathematische Zeitschrift, vol. 29, no. 1, pp. 730-736, 1929.

[12] S. Odake and R. Sasaki, "Infinitely many shape invariant discrete quantum mechanical systems and new exceptional orthogonal polynomials related to the Wilson and Askey-Wilson polynomials," Physics Letters B, vol. 682, no. 1, pp. 130-136, 2009.

[13] S. Odake and R. Sasaki, "The Exceptional $\left(X_{\ell}\right)(q)$-Racah Polynomials," Progress of Theoretical Physics, vol. 125, no. 5, pp. 851-870, 2011.

[14] D. Gómez-Ullate, N. Kamran, and R. Milson, "Exceptional orthogonal polynomials and the Darboux transformation," Journal of Physics A, vol. 43, no. 43, Article ID 434016, 16 pages, 2010.

[15] R. Sasaki, S. Tsujimoto, and A. Zhedanov, "Exceptional Laguerre and Jacobi polynomials and the corresponding potentials through Darboux-Crum transformations," Journal of Physics A, vol. 43, no. 31, Article ID 315204, 20 pages, 2010.

[16] C. L. Ho, "Dirac(-Pauli), Fokker-Planck equations and exceptional Laguerre polynomials," Annals of Physics, vol. 326, no. 4, pp. 797-807, 2011.

[17] G. Szego, Orthogonal Polynomials, vol. 23 of American Mathematical Society Colloquium Publications, American Mathematical Society, New York, NY, USA, 1939.

[18] T. S. Chihara, An Introduction to Orthogonal Polynomials, vol. 13 of Mathematics and its Applications, Gordon and Breach Science, New York, NY, USA, 1978.

[19] G. E. Andrews, R. Askey, and R. Roy, Special Functions, vol. 71 of Encyclopedia of Mathematics and its Applications, Cambridge University Press, Cambridge, UK, 1999.

[20] M. E. H. Ismail, Classical and Quantum Orthogonal Polynomials in One Variable, vol. 98 of Encyclopedia of Mathematics and its Applications, Cambridge University Press, Cambridge, UK, 2005.

[21] Y. Grandati and A. Bérard, "Solvable rational extension of translationally shape invariantpotentials," http://128.84.158.119/abs/0912.3061v2.

[22] Y. Grandati, "Solvable rational extensions of the isotonic oscillator," Annals of Physics, vol. 326, no. 8, pp. 2074-2090, 2011.

[23] Y. Grandati, "Solvable rational extensions of the Morse and Kepler-Coulomb potentials," Journal of Mathematical Physics, vol. 52, no. 10, Article ID 103505, 12 pages, 2011. 


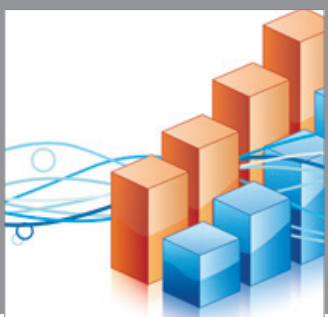

Advances in

Operations Research

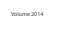

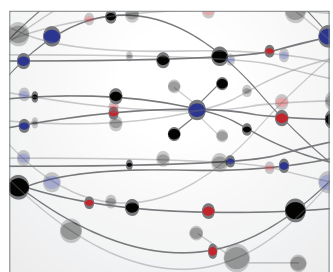

\section{The Scientific} World Journal
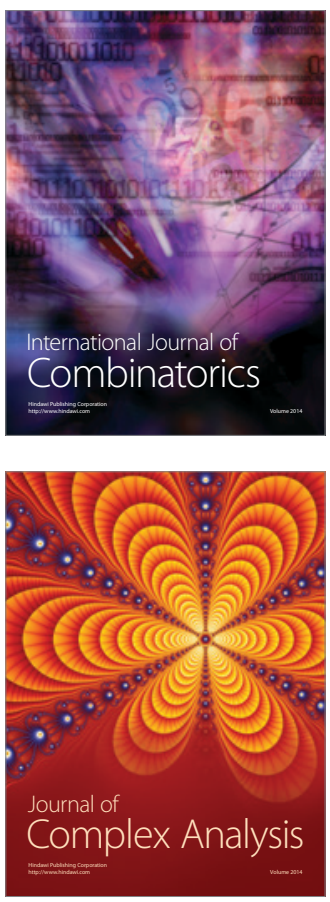

International Journal of

Mathematics and

Mathematical

Sciences
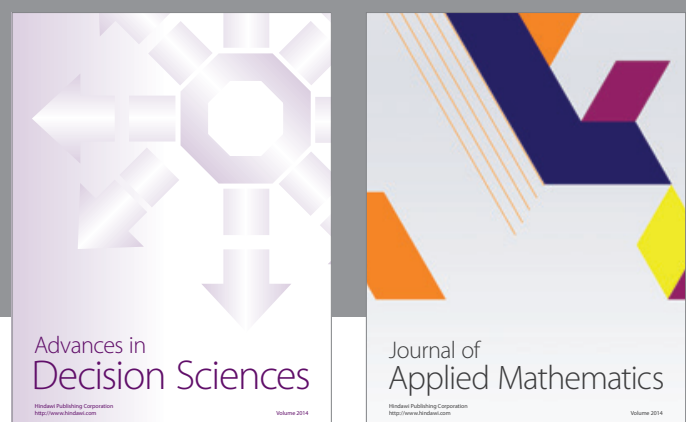

Journal of

Applied Mathematics
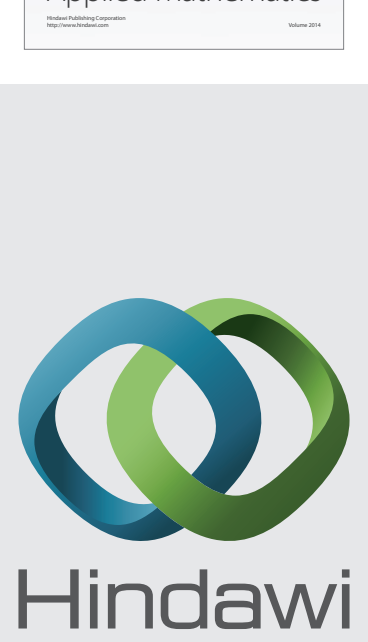

Submit your manuscripts at http://www.hindawi.com
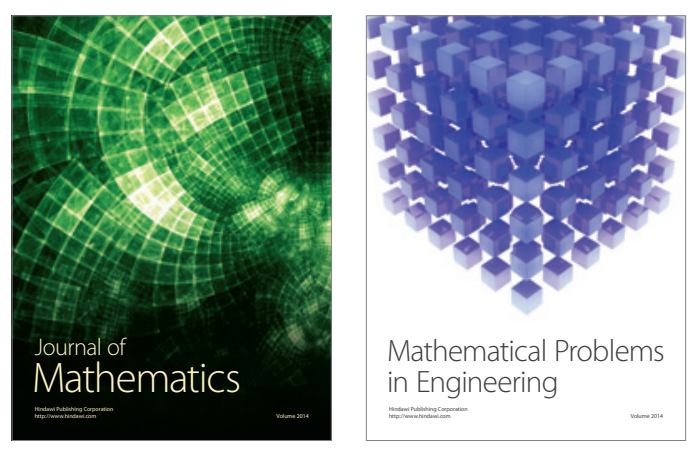

Mathematical Problems in Engineering
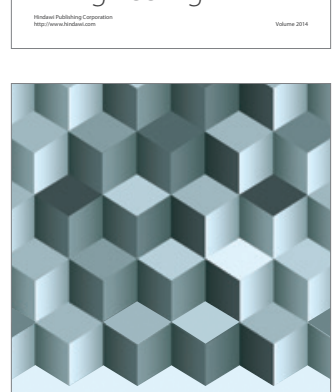

Journal of

Function Spaces
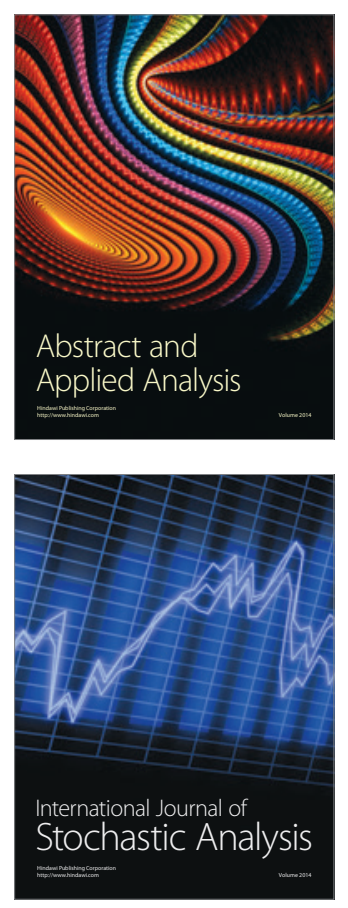

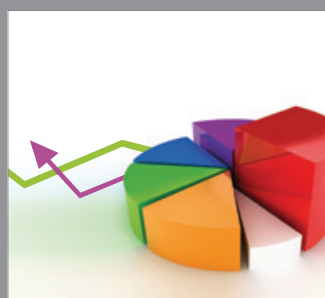

ournal of

Probability and Statistics

Promensencen
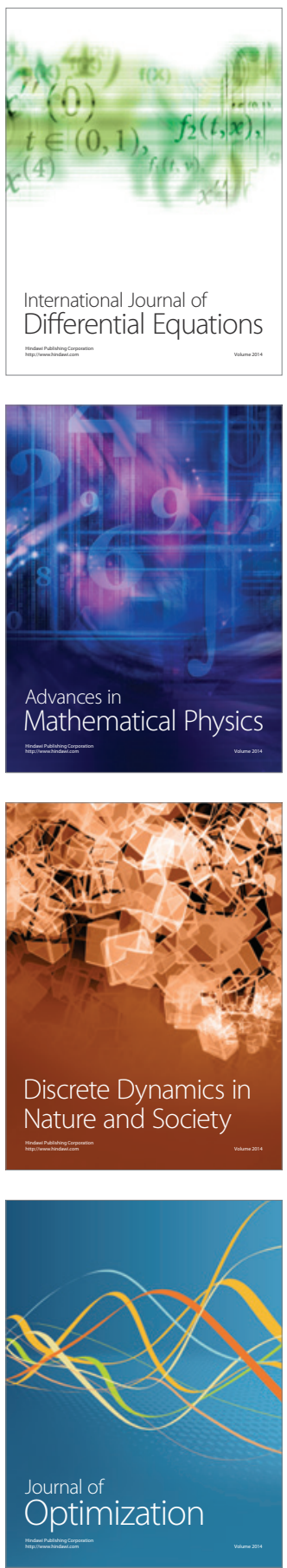